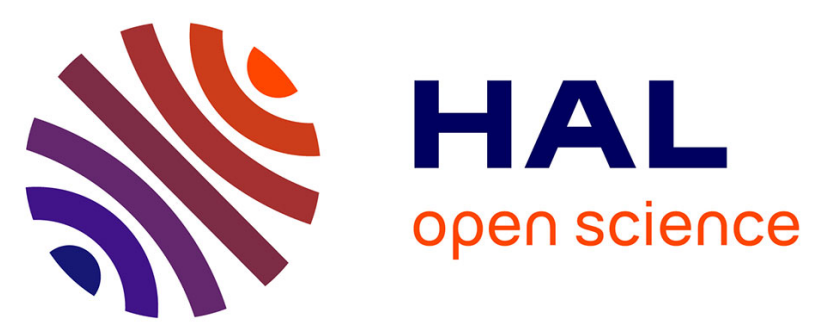

\title{
Résonance magnétique en champ de radiofréquence inhomogène. 1 re partie: Étude théorique
}

\author{
René Barbé, M. Leduc, F. Laloë
}

\section{To cite this version:}

René Barbé, M. Leduc, F. Laloë. Résonance magnétique en champ de radiofréquence inhomogène. 1 re partie: Étude théorique. Journal de Physique, 1974, 35 (10), pp.699-725. 10.1051/jphys:019740035010069900 . jpa-00208198

\section{HAL Id: jpa-00208198 https://hal.science/jpa-00208198}

Submitted on 1 Jan 1974

HAL is a multi-disciplinary open access archive for the deposit and dissemination of scientific research documents, whether they are published or not. The documents may come from teaching and research institutions in France or abroad, or from public or private research centers.
L'archive ouverte pluridisciplinaire HAL, est destinée au dépôt et à la diffusion de documents scientifiques de niveau recherche, publiés ou non, émanant des établissements d'enseignement et de recherche français ou étrangers, des laboratoires publics ou privés. 


\title{
RÉSONANCE MAGNÉTIQUE EN CHAMP DE RADIOFRÉQUENCE INHOMOGĖNE. $1^{\text {re }}$ partie : ÉTUDE THÉORIQUE
}

\author{
R. BARBÉ, M. LEDUC et F. LALOË \\ Laboratoire de Spectroscopie Hertzienne de l'E. N. S., 24, rue Lhomond, 75231 Paris Cedex 05
}

(Reçu le 26 mars 1974)

\begin{abstract}
Résumé. - On étudie dans cet article l'effet d'un champ de radiofréquence très inhomogène sur un ensemble de spins en mouvement. Lorsque le champ magnétique statique est balayé au voisinage de la résonance, les variations de l'aimantation longitudinale globale des spins donnent des courbes de résonance nettement différentes des courbes habituelles; elles sont en général la superposition de deux courbes de largeurs très différentes, la courbe large et la courbe fine. La forme de la première dépend essentiellement des caractéristiques du mouvement des atomes dans le champ de radiofréquence inhomogène. On étudie en détail le cas où ce mouvement est régi par un processus de diffusion : l'étude de la largeur à mi-hauteur de la courbe large donne alors le temps de diffusion des atomes dans la cellule, ce qui fournit un procédé commode de mesure des coefficients de diffusion dans les gaz. La courbe fine peut avoir, suivant les variations spatiales des inhomogénéités statique et de radiofréquence (et notamment suivant les corrélations entre les différentes composantes de ces inhomogénéités) des formes variées : elle peut être constituée d'une courbe d'absorption positive ou négative (aimantation minimale ou maximale à résonance), ou de la superposition d'une courbe d'absorption et d'une courbe de dispersion. De plus, des déplacements importants de la position de la résonance fine sont possibles, notamment sous l'effet de termes croisés entre inhomogénéités statiques et de radiofréquence.
\end{abstract}

\begin{abstract}
The effect of a very inhomogeneous radiofrequency field on an ensemble of moving atoms is investigated theoretically. When sweeping the static magnetic field and measuring the longitudinal magnetization, one observes a resonance curve of unusual shape. In most cases it is the superposition of two curves differing substantially in width. The shape of the broad curve depends primarily on the atomic motion in the inhomogeneous radiofrequency field. The case in which the motion follows a diffusion process is investigated in detail and the half-width of the broad curve is then simply related to the diffusion time of the atoms through the cell. This yields a sensitive method for measuring diffusion coefficients in gases without requiring determination of the magnitude of the field gradient. The narrow curve may have various shapes according to the spatial variations of the static and radiofrequency field inhomogeneities (and mainly to the correlations between the different components of such inhomogeneities). The curve may have an absorption shape, with a positive or a negative sign (the magnetization being minimum or maximum at resonance) and may also be a superposition of an absorption and a dispersion curve. Furthermore, under certain conditions, the narrow curve undergoes large shifts, in particular due to crossed terms between the static and radiofrequency field inhomogeneities.
\end{abstract}

Le principe général des expériences de résonance magnétique est bien connu [1]: on considère un ensemble d'atomes, dont chacun possède un moment cinétique $\mathbf{S}$ et un moment magnétique $\mathbf{m}=\gamma \mathbf{S}$ $(\gamma:$ rapport gyromagnétique des atomes) qu'un processus quelconque (relaxation thermique, pompage optique, etc.) tend à orienter dans une direction $O z$; ces atomes sont plongés dans un champ statique $\mathbf{B}_{0}$ parallèle à $O z$ et soumis à l'action d'un second champ magnétique $\mathbf{B}_{1}(t)$ (champ de radiofréquence), tournant autour de $O z$ avec la vitesse angulaire $\omega$; lorsque $B_{0} \simeq-\omega / \gamma$, il se produit un phénomène de résonance (résonance magnétique) : le champ de radiofréquence, même faible, peut détruire l'orientation des atomes. Les variations en fonction de $B_{0}$ de la composante $M_{z}$ sur $O z$ de $\mathbf{M}$ (aimantation globale de l'ensemble des atomes) sont données par une courbe d'absorption de Lorentz, centrée en $B_{0}=-\omega / \gamma$, et dont la largeur dépend de $B_{1}$ ainsi que des temps de relaxation longitudinale et transversale du système.

En pratique, les champs $\mathbf{B}_{0}$ et $\mathbf{B}_{1}$ ne sont évidemment jamais parfaitement homogènes, et il se pose le problème de tenir compte de cette inhomogénéité. 
Si chaque atome est parfaitement immobile, on peut simplement calculer l'évolution de son moment magnétique à partir du champ local $\mathbf{B}_{0}(\mathbf{r})$ et $\mathbf{B}_{1}(\mathbf{r}, t)$ qu'il voit, quitte à sommer ensuite sur les différents atomes pour obtenir l'aimantation globale M. En fait, dans un grand nombre d'expériences, les atomes se déplacent sous l'effet de l'agitation thermique et, par l'intermédiaire de leur position $\mathbf{r}$, les valeurs des champs $\mathbf{B}_{0}$ et $\mathbf{B}_{1}$ auxquels ils sont soumis sont des fonctions aléatoires du temps. C'est d'ailleurs surtout l'effet des inhomogénéités du champ $\mathbf{B}_{0}$ qui est susceptible de se manifester car, le champ statique étant généralement bien plus intense que le champ de radiofréquence, ses variations sur l'échantillon étudié peuvent être beaucoup plus grandes. Ainsi l'effet des inhomogénéités de $\mathbf{B}_{0}$ a-t-il été fréquemment étudié ; par exemple, dans le cas où le mouvement des atomes est suffisamment rapide (condition de rétrécissement par le mouvement [1] [2]), ces inhomogénéités se traduisent simplement par un raccourcissement des temps de relaxation exponentielle de $\mathbf{M}$ [3] [4] [5] [6] et, par suite, par un élargissement de la courbe de Lorentz donnant la résonance. Des déplacements de la fréquence de résonance sont également possibles mais, sauf dans certains cas particuliers (voir par exemple [7]), ils restent faibles devant la largeur de raie. Toutefois, bien que souvent faible, l'effet des inhomogénéités de $\mathbf{B}_{1}$ n'est pas toujours négligeable, surtout dans les expériences où, pour obtenir une puissance importante de radiofréquence, on utilise des bobines de petite taille pour créer $\mathbf{B}_{1}$; il existe d'ailleurs des types d'expériences où, précisément pour éliminer les effets des inhomogénéités de $\mathbf{B}_{0}$, on utilise un champ $B_{1}$ très intense [1] [8] [9] $\left(B_{1}\right.$ doìt être supérieur aux inhomogénéités de $B_{0}$ sur la cellule). Ce sont alors les inhomogénéités de $B_{1}$ qui deviennent gênantes et, pour s'en affranchir, on utilise quelquefois la technique des rotary echoes [10] ou échos de spin dans le référentiel tournant.

Les inhomogénéités du champ de radiofréquence dans une expérience de résonance magnétique peuvent donc dans certains cas jouer un rôle important; c'est ce rôle que nous allons étudier ici. Nous allons d'emblée considérer un cas extrême, celui où la radiofréquence est complètement inhomogène (à tout instant, la valeur moyenne de $\mathbf{B}_{1}$ sur la cellule renfermant les atomes est nulle) ; le cas où $\mathbf{B}_{1}$ comprend également une partie homogène sera examiné ensuite. Nous nous limiterons cependant toujours au cas où le champ $\mathbf{B}_{1}$ est suffisamment faible (ou le mouvement des atomes dans la cellule suffisamment rapide) pour que son action soit soumise au « rétrécissement par le mouvement ", comme c'est très fréquemment le cas en pratique (au moins dans les expériences de résonance magnétique sur des échantillons gazeux). L'hypothèse du rétrécissement par le mouvement permet de mener les calculs jusqu'au bout en caractérisant l'orientation des atomes par une aimantation globale M, sans avoir besoin d'introduire une aiman- tation $\mathbf{M}(\mathbf{r})$ dépendant de $\mathbf{r}$ [11]. Nous verrons alors que l'effet des inhomogénéités de la radiofréquence peut être plus radical qu'un simple changement des caractéristiques (largeur, hauteur) de la courbe de résonance. De fait, cette courbe apparaîtra comme la superposition d'une courbe d'absorption large (souvent beaucoup plus large que celle de résonance magnétique habituelle) et d'une courbe fine (de largeur comparable à la largeur habituelle) dont la forme peut, suivant les conditions expérimentales, être plus ou moins compliquée : elle peut se présenter comme une courbe d'absorption, positive ou négative $\left(M_{z}\right.$ est minimal ou maximal à résonance), ou encore comme une superposition d'une courbe d'absorption et d'une courbe de dispersion. De plus, des déplacements notables (comparables à la largeur) de la courbe fine sont possibles. D'un point de vue pratique, de tels déplacements peuvent être importants pour certaines expériences de haute résolution : si le champ de radiofréquence n'est pas parfaitement homogène, la faible composante inhomogène peut produire une courbe large peu visible dans l'expérience (elle apparaîtra comme une légère remontée $\mathrm{du}$ fond continu) ; cependant, lors du pointé de la résonance fine, le fait qu'elle soit déplacée et, de plus, comprenne une courbe de dispersion (courbe asymétrique) peut conduire à des erreurs. Une autre application pratique de notre étude provient de ce que les paramètres associés à la courbe large (forme et largeur) dépendent de façon critique du mouvement des atomes dans la cellule; dans le cas où ce mouvement obéit à l'équation de la diffusion, l'étude expérimentale de ces caractéristiques peut conduire à des mesures de coefficients de diffusion.

Dans ce premier article, nous étưđierons théoriquement l'effet des inhomogénéités du champ de radiofréquence sur l'aimantation $\mathbf{M}$ de l'ensemble des atomes. Un premier modèle simple ( $\S$ A) montrera que l'effet de la partie inhomogène se traduit par l'existence d'un processus de relaxation résonnante agissant sur les atomes. Les variations en fonction de $B_{0}$ du temps de relaxation longitudinale correspondant permettent de comprendre l'existence de la courbe large. Nous reprendrons ensuite $\left(\begin{array}{l}\S \\ \text { B }\end{array}\right)$ le problème de façon plus précise et calculerons en détail les équations d'évolution des composantes de $\mathbf{M}$, compte tenu de l'existence de plusieurs modes de diffusion dans la cellule sphérique, du fait que la carte du champ inhomogène sur cette cellule peut être plus ou moins complexe, etc. Pour traiter le cas le plus fréquent en pratique, nous envisagerons celui où les champs statique et oscillant sont tous deux inhomogènes. Enfin $(\S \mathrm{C})$, nous calculerons la forme de la courbe de résonance donnant les variations de $M_{z}$ en fonction de $B_{0}$; en particulier, dans le cas où $\mathbf{B}_{0}$ et $\mathbf{B}_{1}$ sont tous deux inhomogènes, nous étudierons l'influence de la relaxation résonnante anisotrope correspondante sur la forme plus ou moins complexe de la courbe fine.

Un second article, faisant suite à celui-ci, rendra 
compte des vérifications détaillées de ces calculs, effectuées au moyen d'une expérience d'orientation nucléaire de ${ }^{3} \mathrm{He}$ gazeux obtenue par pompage optique. Signalons qu'un bref résumé des résultats théoriques et expérimentaux de ces deux articles est donné dans les références [12] et [13]. Par ailleurs, des expériences de pompage optique sur ${ }^{201} \mathrm{Hg}$ [14] et ${ }^{87} \mathrm{Rb}[15]$ ont permis d'observer des effets analogues sur des atomes autres que l'hélium.

A. Modèle simple. - A.1 Champ DE RAdiofréQUENCE PARFAITEMENT INHOMOGÈNE ; COURBE LARGE.Nous supposons ici que la cellule contenant les atomes est plongée dans un champ statique $\mathbf{B}_{0}$, parallèle à l'axe $O z$, d'intensité

$$
B_{0}=-\frac{\omega_{0}}{\gamma}
$$

Les atomes sont également soumis à l'action d'un champ inhomogène oscillant $\mathbf{B}_{1}(\mathbf{r}, t)$ que nous appellerons champ de radiofréquence :

$$
\mathbf{B}_{1}(\mathbf{r}, t)=-\frac{\mathbf{\Omega}_{1}(\mathbf{r})}{\gamma} \cos \omega t
$$

où $\boldsymbol{\Omega}_{1}(\mathbf{r})$ est une fonction vectorielle donnée variant d'un point $\mathbf{r}$ à un autre dans la cellule. La valeur moyenne sur la cellule, de volume $V$, s'écrit :

$$
\left\langle\boldsymbol{\Omega}_{1}(\mathbf{r})\right\rangle=\int \boldsymbol{\Omega}_{1}(\mathbf{r}) \frac{\mathrm{d}^{3} r}{V} .
$$

Nous allons commencer par supposer dans cet article que $\left\langle\boldsymbol{\Omega}_{1}(\mathbf{r})\right\rangle=0$ (champ de radiofréquence parfaitement inhomogène); par contre, bien évidemment :

$$
\left\langle\boldsymbol{\Omega}_{1}^{2}(\mathbf{r})\right\rangle \neq 0 .
$$

$\mathrm{Du}$ fait de son mouvement d'agitation thermique, chaque atome du gaz voit un champ qui dépend du temps à la fois explicitement et implicitement, la dépendance implicite venant des variations aléatoires de sa position r. Pour comprendre l'effet sur les atomes du champ de radiofréquence, il est utile d'utiliser le référentiel tournant (référentiel tournant autour de $O z$ à la vitesse angulaire $\omega)\left({ }^{1}\right)$. Le champ de radiofréquence devient alors un champ statique, à condition de négliger en chaque point $\mathbf{r}$ de la cellule la composante tournante antirésonnante de $\mathbf{B}_{1}(\mathbf{r}, t)$ ainsi que sa partie oscillante parallèle à $O z$; cette approximation est valable lorsque l'intensité du champ $\mathbf{B}_{0}$ n'est pas trop faible (il faut notamment que $\omega_{0} \gg \Omega_{1}(\mathbf{r})$ ), ce qui est le cas en pratique dans la plupart des expériences. Nous sommes alors

( $\left.{ }^{1}\right)$ On prend en fait en chaque point $\mathbf{r}$ un référentiel tournant, dont l'origine reste au point fixe $\mathbf{r}$, et animé d'une rotation de vitesse angulaire $\omega$. En d'autres termes, on effectue en chaque point une transformation sur les composantes de $\mathbf{M}$ et de $\mathbf{B}_{1}$, mais pas de $\mathbf{r}$. ramenés à un problème bien connu : celui de l'effet d'un champ statique inhomogène sur une collection d'atomes en mouvement thermique ; on sait ([3] à [6]) que l'existence d'un tel champ se traduit pour les atomes par une relaxation supplémentaire de leur aimantation globale. Nous allons supposer ici que la pression dans la cellule est telle que le mouvement des atomes peut être traité classiquement et qu'il obéit à l'équation de diffusion $\left({ }^{2}\right)$. Désignons par $\tau_{d}$ le temps de diffusion des atomes dans la cellule (la définition précise de $\tau_{\mathrm{d}}$ sera donnée dans le $\S$ B. 2 suivant) ; $\tau_{d}$ est le temps de corrélation de la perturbation magnétique aléatoire agissant sur les atomes. Lorsque :

$$
\left\langle\Omega_{1}^{2}(\mathbf{r})\right\rangle \tau_{\mathrm{d}}^{2} \ll 1
$$

(condition de rétrécissement par le mouvement [1] [2] [16]), le temps de relaxation $T_{1}^{*}$ de la composante longitudinale $M_{z}$ de l'aimantation globale $\mathbf{M}$ des atomes est donné par $\left({ }^{3}\right)$ :

$$
\frac{1}{T_{1}^{*}}=\frac{\left(\left\langle\Omega_{1 \perp}^{2}\right\rangle / 4\right) \tau_{\mathrm{d}}}{1+\left(\Delta_{0} \tau_{\mathrm{d}}\right)^{2}}
$$

où :

$$
\Delta_{0}=\omega_{0}-\omega
$$

et où :

$$
\left\langle\Omega_{1 \perp}^{2}\right\rangle=\left\langle\Omega_{1 x}^{2}(\mathbf{r})+\Omega_{1 y}^{2}(\mathbf{r})\right\rangle
$$

est la valeur moyenne sur la cellule du carré de la projection transversale du champ de radiofréquence inhomogène (physiquement, il est évident que la composante longitudinale $\Omega_{1 z}(\mathrm{r})$ n'agit pas sur $\left.M_{z}\right)$. La formule (A.6) est classique ; on notera seulement que c'est $\Delta_{0}$ qui apparaît à son dénominateur, et non $\omega_{0}$ comme habituellement; ceci tient tout simplement au fait que nous raisonnons ici dans le référentiel tournant où l'intensité du champ statique efficace le long de $O z$ est proportionnelle à $\Delta_{0}$ (et non à $\omega_{0}$ ). La probabilité de relaxation $1 / T_{1}^{*}$ a donc un comportement résonnant dans la région $\Delta_{0} \simeq 0$, et devient beaucoup moins importante dès que $\left|\Delta_{0}\right| \gg 1 / \tau_{\mathrm{d}}$; physiquement, ceci tient au fait que la perturbation aléatoire magnétique dans le référentiel tournant possède une densité spectrale notable dans une bande de fréquence centrée en zéro et de largeur comparable à $1 / \tau_{\mathrm{d}}$, la fréquence propre du spin dans ce référentiel étant $\left|\Delta_{0}\right| / 2 \pi$.

L'effet d'un champ de radiofréquence parfaitement inhomogène est donc très notablement différent de celui d'un champ homogène; au lieu de coupler entre elles les composantes longitudinale $M_{z}$

( ${ }^{2}$ ) Aux faibles pressions où le libre parcours moyen des atomes serait comparable ou supérieur aux dimensions de la cellule, cette hypothèse ne serait pas valable.

$\left({ }^{3}\right)$ Dans l'expression (A.6), le facteur $1 / 4$ provient de ce que seule une des deux composantes tournantes du champ de radiofréquence intervient. Il faut d'ailleurs noter que cette expression n'est qu'approchée; la valeur précise de $T_{1}^{*}$ sera calculée au $\S$ B.3.a. $\alpha$. 
et transversale $M_{+}=M_{x}+i M_{y}$, il ne couple $M_{z}$ qu'à $M_{z}$, dont l'évolution globale s'écrit :

$\frac{\mathrm{d} M_{z}}{\mathrm{~d} t}=-M_{z}\left(\frac{1}{T_{\mathrm{r}}}+\frac{1}{T_{1}^{*}}\right)+\frac{1}{T_{\mathrm{p}}}\left(M_{0}-M_{z}\right)$.

Dans cette équation, le terme en $1 / T_{p}$ traduit l'action d'un processus d'orientation longitudinale des atomes (pompage optique par exemple), le terme en $1 / T_{\mathrm{r}}+1 / T_{1}^{*}$ l'effet des autres processus de relaxation $\left(T_{\mathrm{r}}\right.$ désigne le temps de relaxation de $M_{z}$ en l'absence d'inhomogénéités de radiofréquence et du processus d'orientation des atomes). L'orientation $M_{z}$ évolue donc avec une constante de temps globale $T_{1}$ donnée par :

$$
\frac{1}{T_{1}}=\frac{1}{T_{1}^{0}}+\frac{1}{T_{1}^{*}}
$$

où :

$$
\frac{1}{T_{1}^{0}}=\frac{1}{T_{\mathrm{r}}}+\frac{1}{T_{\mathrm{p}}}
$$

désigne la probabilité de relaxation de $M_{z}$ en l'absence de champ de radiofréquence inhomogène. Quant à la valeur stationnaire limite $M_{z}^{\text {s }}$ de $M_{z}$, on l'obtient facilement à partir de (A.6), (A.9) et (A.10) :

$$
\begin{aligned}
M_{z}^{\mathrm{s}}= & M_{0} \frac{T_{1}}{T_{\mathrm{p}}} \\
= & M_{0} \frac{T_{\mathrm{r}}}{T_{\mathrm{r}}+T_{\mathrm{p}}} \times \\
& \times\left[1-\frac{\left(\left\langle\Omega_{1 \perp}^{2}\right\rangle / 4\right) T_{1}^{0} \tau_{\mathrm{d}}}{1+\left(\Delta_{0} \tau_{\mathrm{d}}\right)^{2}+\left(\left\langle\Omega_{1 \perp}^{2}\right\rangle / 4\right) T_{1}^{0} \tau_{\mathrm{d}}}\right] .
\end{aligned}
$$

Comme en résonance magnétique ordinaire, les variations de $M_{z}^{\text {s }}$ sont donc données par une courbe de Lorentz, centrée en $\Delta_{0}=0$, et dont la largeur et la hauteur sont des fonctions croissantes de l'intensité du champ de radiofréquence appliqué. Des différences importantes apparaissent toutefois ; par exemple, le temps de relaxation transversale $T_{2}^{0}$ ne figure pas dans (A.11), ce qui se comprend bien puisque $M_{z}$ n'est pas couplé à $M_{+}$. La différence la plus importante tient cependant à la valeur de la largeur $L$ de la courbe prévue par (A.11) qui vaut, exprimée en unités de pulsations (dans tout cet article nous appelons simplement largeur des courbes leur demi-largeur à mi-hauteur) :

$$
L=\frac{1}{\tau_{\mathrm{d}}} \sqrt{1+\left(\left\langle\Omega_{1 \perp}^{2}\right\rangle / 4\right) T_{1}^{0} \tau_{\mathrm{d}}} .
$$

Cette largeur, extrapolée à intensité de radiofréquence nulle, donne $1 / \tau_{\mathrm{d}}$, et non $1 / T_{2}^{0}$ comme en résonance magnétique ordinaire $\left(T_{2}^{0}\right.$ : temps de relaxation transversale en l'absence de champ R. F. inhomogène). Nous allons supposer ici que :

$$
\tau_{\mathrm{d}} \ll T_{2}^{0} \text {. }
$$

La courbe de résonance donnée par (A.11) est alors beaucoup plus large que celle que donnerait la résonance avec un champ de radiofréquence homogène; c'est pourquoi nous l'appellerons "courbe large".

Remarques :

(i) La formule (A.9) prévoit exactement la même forme de courbe que les formes habituelles de résonance magnétique pour un système dont les temps de relaxation longitudinale et transversale seraient $T_{1}^{0}$ et $\tau_{\mathrm{d}}$.

(ii) Les courbes centrées en $\omega_{0}= \pm \omega$ ne se recouvrent pas si :

$$
\omega \gg L
$$

Dans toute la suite, nous supposerons que :

$$
\begin{aligned}
& \omega \gg 1 / \tau_{\mathrm{d}} \\
& \omega \gg \sqrt{\left\langle\Omega_{1 \perp}^{2}\right\rangle} \sqrt{\frac{T_{1}^{0}}{\tau_{\mathrm{d}}}} \\
& \omega \gg\left|\omega_{0}-\omega\right| .
\end{aligned}
$$

L'effet de la composante antirésonnante est alors négligeable.

A. 2 Champ De RADIOFRÉQUenCE COMPORTANT UNE PARTIE HOMOGÈNE; COURBE FINE. - Que se passe-t-il maintenant si le champ de radiofréquence n'est pas parfaitement inhomogène, c'est-à-dire si $\left\langle\boldsymbol{\Omega}_{1}(\mathbf{r})\right\rangle$ n'est pas nul (ou parallèle à $O z$ ) ? Il est clair que, contrairement à ce qui se produisait au $\S 1$ précédent, les évolutions de $M_{z}$ et de $M_{+}$ne sont plus découplées ; on s'attend donc à une modification de la forme de courbe de résonance.

On peut décomposer tout champ de radiofréquence inhomogène en deux parties : la première

$$
-\frac{1}{\gamma}\left[\mathbf{\Omega}_{1}(\mathbf{r})-\left\langle\mathbf{\Omega}_{1}(\mathbf{r})\right\rangle\right] \cos \omega t
$$

est purement inhomogène ; la seconde

$$
-\frac{1}{\gamma}\left\langle\boldsymbol{\Omega}_{1}(\mathbf{r})\right\rangle \cos \omega t=-\frac{\boldsymbol{\omega}_{1}}{\gamma} \cos \omega t
$$

est au contraire parfaitement homogène et dépend uniquement de la valeur moyenne du champ de radiofréquence, notée pour simplifier :

$$
\omega_{1}=\left\langle\mathbf{\Omega}_{1}(\mathbf{r})\right\rangle .
$$

Le problème est maintenant de savoir dans quelle mesure on peut simplement ajouter l'effet de la partie inhomogène du champ (relaxation résonnante) et celui de sa partie homogène. Dans tout cet article, nous supposerons que :

$$
\omega_{1} \tau_{\mathrm{d}} \ll 1 .
$$

Cette condition, réalisée en pratique dans la plupart des expériences du fait de la petite valeur du temps de diffusion ( $\tau_{d}$ est couramment de l'ordre de $10^{-3} \mathrm{~s}$ ), 
exprime que la partie homogène du champ de radiofréquence n'a pas le temps d'agir pendant le temps de corrélation de la perturbation magnétique aléatoire à laquelle est soumis chaque atome. Dans ce cas, on sait [1] [16] que la relaxation associée à cette perturbation aléatoire n'est pas modifiée par la présence du champ de radiofréquence homogène $\left({ }^{4}\right)$. Par suite, tous les résultats du $\S 1$ précédent concernant l'effet de la partie inhomogène sur l'évolution de l'aimantation $\mathbf{M}$ restent valables. L'équation d'évolution globale de $\mathbf{M}$ s'obtient donc simplement en ajoutant au second membre les termes provenant de la présence du champ de radiofréquence homogène.

Nous sommes donc ramenés à un problème classique de résonance magnétique : calculer l'effet d'un champ de radiofréquence oscillant homogène sur l'aimantation $\mathbf{M}$ d'un ensemble d'atomes, compte tenu bien sûr du fait que la partie inhomogène du champ oscillant produit une relaxation supplémentaire pour $\mathbf{M}$. $\mathrm{Au} \S 1$ précédent, nous avons vu que la modification de la relaxation longitudinale conduit à remplacer $T_{1}^{0}$ par $T_{1}<T_{1}^{0}$ (cf. (A.10a)) ; il faut évidemment envisager également les modifications de la relaxation transversale, et remplacer le temps transversal $T_{2}^{0}$ en l'absence de radiofréquence par un temps $T_{2}$ plus court $\left({ }^{5}\right)$ :

$$
\frac{1}{T_{2}}=\frac{1}{T_{2}^{0}}+\frac{1}{T_{2}^{*}}
$$

$\left(T_{2}^{*}\right.$ : temps de relaxation transversale associé au champ de radiofréquence inhomogène $\left.\left({ }^{6}\right)\right)$. Ces modifications une fois faites, on peut appliquer les classiques formules de Bloch pour obtenir l'effet sur la valeur stationnaire de $\mathbf{M}$ du champ de radiofréquence homogène; on trouve pour la valeur stationnaire $M_{z}^{\text {s }}$ de $M_{z}$ l'expression habituelle (voir par exemple, réf. [1], formule (3) du Chap. III, p. 44) :

$$
M_{z}^{\mathrm{s}}=M_{0} \frac{T_{1}}{T_{\mathrm{p}}}\left\{1-\frac{\omega_{1 \perp}^{2} T_{1} / 4 T_{2}}{\Delta_{0}^{2}+\left(1 / T_{2}\right)^{2}+\omega_{1 \perp}^{2} T_{1} / 4 T_{2}}\right\}
$$

où $\omega_{1 \perp}$ désigne le module de la projection de $\omega_{1}$ sur le plan $x O y$ perpendiculaire à $O z$. Si $T_{1}$ et $T_{2}$ étaient des constantes, cette formule donnerait pour $M_{z}^{\mathrm{s}}$ une courbe de Lorentz de largeur :

$$
l=\frac{1}{T_{2}} \sqrt{1+\omega_{1 \perp}^{2} T_{1} T_{2} / 4}
$$

$\left.{ }^{4}\right) \mathrm{La}$ condition (A.18) est discutée dans la référence [17] (Chap. VII) en termes d'atomes habillés par le champ de radiofréquence (elle exprime que les modifications des fréquences de Bohr sont faibles devant la largeur spectrale de la perturbation aléatoire).

$\left({ }^{5}\right)$ La relaxation produit également un déplacement $\delta \omega_{0}^{*}$ de la fréquence propre de $M_{+}$; ce déplacement sera étudié au § B.3.a. $\beta$ suivant.

$\left(^{6}\right)$ La valeur exacte de $T_{2}^{*}$ sera précisée plus bas (§ B.3.a. $\left.\beta\right)$; signalons dès ici que $T_{2}^{*}$ est comparable à $T_{1}^{*}$.
En fait, $T_{1}$ et $T_{2}$ dépendent de $\Delta_{0}$ par l'intermédiaire de $T_{1}^{*}$ et $T_{2}^{*}$ (voir par exemple (A.6) et (A.10)). Cependant leurs variations ne sont notables que si $\Delta_{0}$ varie d'une quantité de l'ordre de $1 / \tau_{\mathrm{d}}$; nous allons supposer ici que :

$$
l \ll \frac{1}{\tau_{\mathrm{d}}}
$$

hypothèse qui, sauf pour les très grandes valeurs du champ de radiofréquence homogène (résonance très, fortement saturée) revient à :

$$
T_{2}^{0} \gg \tau_{\mathrm{d}} \text {. }
$$

Dans ces conditions, les variations de $T_{1}$ et $T_{2}$ sur la largeur $l$ sont complètement négligeables et on peut les remplacer dans (A.20) par leurs valeurs pour $\Delta_{0}=0$; cette formule prévoit alors pour la valeur stationnaire $M_{z}^{s}$ de l'orientation longitudinale une variation lorentzienne dans la région $\left|\Delta_{0}\right| \lesssim l$.

Globalement, la courbe de résonance est donc la somme de deux courbes d'absorption lorentziennes (cf. Fig. 1). Loin de la résonance $\left(\left|\Delta_{0}\right| \gg l\right)$, l'effet du champ de radiofréquence homogène est négligeable, et la courbe de résonance est confondue avec la courbe large donnée par (A.11). L'effet du champ de radiofréquence homogène n'est sensible (comme habituellement en résonance magnétique) que si $\left|\Delta_{0}\right| \lesssim l$; lorsque cette condition est réalisée, la partie homogène du champ de radiofréquence peut, suivant son intensité, détruire une proportion plus ou moins grande de l'orientation $M_{0} . T_{1}\left(\Delta_{0}=0\right) / T_{\mathrm{p}}$

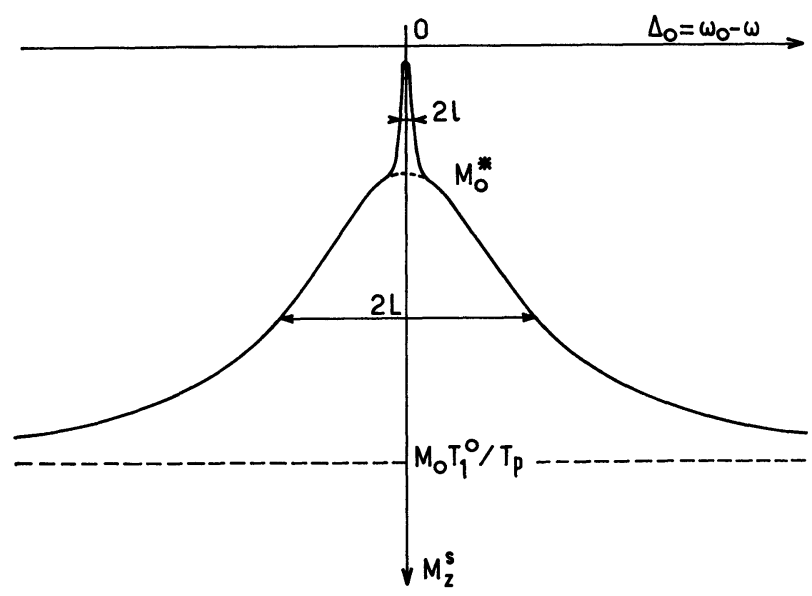

Fig. 1. - Forme de la courbe de résonance obtenue avec un champ de radiofréquence partiellement inhomogène; on porte en fonction de $\Delta_{0}=\omega_{0}-\omega$ les variations de la valeur stationnaire $M_{z}^{\mathrm{s}}$ de l'aimantation longitudinale des atomes. $M_{0} T_{1}^{0} / T_{\mathrm{p}}$ est l'orientation des atomes en l'absence de radiofréquence (on suppose ici $M_{0}>0$ ). La partie inhomogène de ce champ donne des variations résonnantes du temps $T_{1}$ de relaxation longitudinale des atomes, ce qui entraîne l'existence d'une courbe large de largeur $L$ (l'extrapolation de $L$ à radiofréquence nulle donne l'inverse du temps de diffusion des atomes). La partie homogène donne une courbe fine, située au sommet de la courbe large, et de largeur $l$; cette courbe fine peut se décrire comme une résonance magnétique habituelle, au cours de laquelle est détruite une partie de l'orientation $M_{0}^{*}=M_{0} T_{1}\left(\Delta_{0}=0\right) / T_{\mathrm{p}}$ qui aurait subsisté au sommet de la courbe large. 
qui aurait subsisté au sommet de la courbe large ; il en résulte une courbe de résonance fine, superposée à la précédente $\left({ }^{7}\right)$.

A.3 DisCuSSION : DANS QUELLES CONDITIONS EXPÉRIMENTALES LA COURBE LARGE APPARAÎT-ELLE ?Dans une expérience où le champ de radiofréquence est homogène il faut, pour produire la résonance fine, un champ d'intensité telle que :

$$
\omega_{1 \perp}^{2} \gtrsim \frac{1}{T_{1}^{0} T_{2}^{0}} \text {. }
$$

Par contre, si le champ de radiofréquence est parfaitement inhomogène, la résonance large apparaît lorsque :

$$
\left\langle\Omega_{1 \perp}^{2}\right\rangle \gtrsim \frac{1}{T_{1}^{0} \tau_{\mathrm{d}}} .
$$

Comme $T_{2}^{0} \gg \tau_{\mathrm{d}}$, l'intensité du champ oscillant nécessaire pour observer une résonance est plus faible (d'un facteur $\sqrt{\tau_{\mathrm{d}} / T_{2}^{0} \text { ) }}$ lorsque ce champ est homogène que lorsqu'il est inhomogène. On peut alors se poser la question suivante : dans les expériences réalisées en pratique où le champ n'est, ni parfaitement homogène, ni parfaitement inhomogène, l'effet de la composante homogène du champ de radiofréquence ne suffira-t-il pas dans la plupart des cas à masquer celui de la composante inhomogène ? En d'autres termes, si l'on désire observer la résonance large, avec quelle précision faut-il annuler la valeur moyenne du champ oscillant sur la cellule?

Désignons par $k$ un coefficient caractérisant l'inhomogénéité du champ de radiofréquence :

$$
k=\frac{\sqrt{\left\langle\Omega_{1 \perp}^{2}\right\rangle}}{\omega_{1 \perp}}
$$

$k$ varie entre 0 (champ parfaitement homogène) et l'infini (champ de radiofréquence nul en valeur moyenne sur la cellule). Pour que l'on puisse distinguer la courbe large de la courbe fine il faut que, lorsque l'intensité du champ de radiofréquence est assez grande pour qu'apparaisse la courbe large, la courbe fine soit encore suffisamment peu élargie pour rester nettement plus étroite que la courbe large. Or la courbe large apparaît lorsque :

$$
\omega_{1 \perp} \simeq \frac{1}{k \sqrt{T_{1}^{0} \tau_{\mathrm{d}}}}
$$

( ${ }^{7}$ ) On peut se demander si la condition (A.22b) est réellement essentielle et s'il ne suffit pas, pour s'en affranchir, de remplacer dans (A.20) $T_{1}$ et $T_{2}$ par leurs expressions complètes sans négliger leur dépendance en $\Delta_{0}$. En fait, cette manière de faire ne serait pas correcte : pour écrire (A.6), nous avons supposé implicitement que, parmi les processus d'évolution des atomes, seule l'évolution due au champ magnétique statique est capable d'agir notablement sur les atomes en un temps $\tau_{\mathrm{d}}$. De même que la condition (A.18) est nécessaire pour assurer que l'action du champ de radiofréquence homogène est négligeable pendant le temps $\tau_{\mathrm{d}}$, de même il faut pour que (A.6) reste valable qu'aucun processus de relaxation n'agisse efficacement sur l'atome pendant le même temps, ce qui implique nécessairement (A.22b).
Pour cette intensité de radiofréquence, la largeur de la courbe étroite est, d'après (A.21) :

$$
l \simeq \frac{1}{T_{2}^{0}} \sqrt{1+T_{2}^{0} / 4 k^{2} \tau_{\mathrm{d}}} .
$$

Compte tenu de (A.13), on montre facilement que cette largeur est très inférieure à $\tau_{\mathrm{d}}^{-1} \mathrm{si}$

$$
k \gg \sqrt{\frac{\tau_{\mathrm{d}}}{T_{2}^{0}}} .
$$

Donc, pour observer nettement l'effet des inhomogénéités du champ de radiofréquence et obtenir une courbe ayant l'allure de celle de la figure 1 , il faut que l'inhomogénéité du champ de radiofréquence, repérée par le paramètre $k$, soit supérieure à une quantité qui ne dépend que de $\tau_{\mathrm{d}}$ et du temps de relaxation transversale $T_{2}^{0}$. D'après $(\mathrm{A} .22 b)$, cette condition n'est pas très sévère; si l'on pousse suffisamment l'intensité du champ de radiofréquence, la courbe large doit apparaître en fait dans la plupart des expériences.

Remarque. - Compte tenu de (A.26), l'inégalité (A.18) devient

$$
k \gg \sqrt{\frac{\tau_{\mathrm{d}}}{T_{1}^{0}}}
$$

condition qui est, en général, satisfaite si (A.28) l'est.

B. Calcul complet : effets du champ inhomogène sur l'orientation. - Etudions plus en détail l'effet du champ de radiofréquence inhomogène sur l'aimantation des atomes (dans tout ce paragraphe $B$, nous n'étudierons que le rôle de la partie inhomogène du champ de radiofréquence, dont nous supposerons que la valeur moyenne sur la cellule est nulle). Les calculs que nous avons menés jusqu'ici ne sont valables que dans le cadre d'un modèle simple, où l'effet du champ de radiofréquence peut être caractérisé par un temps de relaxation $T_{1}^{*}$ dont l'expression est donnée en (A.6). En fait, la valeur exacte du temps de relaxation longitudinale est plus complexe; elle fait intervenir plusieurs temps de corrélation $\tau_{\mathrm{d}}$ associés aux différents modes de diffusion dans la cellule, avec des poids qui dépendent des variations spatiales du champ de radiofréquence. De plus, il n'est pas suffisant de décrire le processus de relaxation résonnante étudié par un temps de relaxation longitudinale $T_{1}^{*}$, même en lui adjoignant un second temps $T_{2}^{*}$ pour décrire la relaxation transversale : d'une part, la relaxation peut également introduire des déplacements de la fréquence de précession de l'aimantation; d'autre part, elle peut présenter un caractère anisotrope et coupler entre elles différentes composantes de l'aimantation, de sorte que sa description complète nécessite l'introduction d'une matrice $3 \times 3$ de relaxation $(R)$. Nous verrons plus bas ( $\S \mathrm{C}$ et deuxième partie de cet article) 
que ces propriétés complexes de la relaxation (anisotropie, déplacements de fréquence) peuvent conduire à des effets importants et facilement observables. C'est pourquoi nous allons reprendre les calculs du $\S$ A de façon plus détaillée et complète.

B. 1 EVolution Des COMPosantes De L'AimantaTION DANS L'HYPOTHÈSE DU RÉTRÉCISSEMENT PAR LE MOUVEMENT. - Calculons l'effet sur la matrice densité de l'ensemble des atomes de la perturbation magnétique aléatoire que subit chacun d'entre eux, du fait de son mouvement d'agitation thermique dans le gradient de champ magnétique (contrairement au $\S$ A précédent, nous allons ici raisonner dans le référentiel du laboratoire). Nous supposons que les atomes étudiés ont un spin $\frac{1}{2}$ et désignons par $\sigma_{x}, \sigma_{y}$ et $\sigma_{z}$ les opérateurs dans l'espace des états d'un spin $\frac{1}{2}$ associé aux trois matrices de Pauli. L'hamiltonien $\operatorname{Fe}(t)$ de couplage entre un atome, de position $\mathbf{r}(t)$ à l'instant $t$, et le champ de radiofréquence inhomogène s'écrit alors :

$$
\begin{aligned}
\mathscr{H}(t)= & \frac{\hbar}{2}\left\{\Omega_{1 z}[\mathbf{r}(t)] \sigma_{z}+\right. \\
& \left.+\frac{\Omega_{1+}[\mathbf{r}(t)] \sigma_{-}+\Omega_{1-}[\mathbf{r}(t)] \sigma_{+}}{2}\right\} \cos \omega t
\end{aligned}
$$

où $\Omega_{1 \pm}=\Omega_{1 x} \pm i \Omega_{1 y}$ et $\Omega_{1 z}$ sont les composantes $\mathrm{du}$ vecteur $\boldsymbol{\Omega}_{1}(\mathbf{r})$ caractérisant, d'après (A.2), le champ $\mathbf{B}_{1}(\mathbf{r}, t)$; on a posé $\sigma_{ \pm}=\sigma_{x} \pm i \sigma_{y}$. Nous allons supposer ici satisfaite l'hypothèse selon laquelle l'action de $\operatorname{He}(t)$ est soumise au "rétrécissement par le mouvement " [1] [2] [16] :

$$
\left|\boldsymbol{\Omega}_{1}(\mathbf{r})\right| \cdot \tau_{\mathrm{c}} \ll 1
$$

(cette condition étant vérifiée en tout point $\mathbf{r}$ intérieur à la cellule); $\tau_{\mathrm{c}}$ désigne le (ou les) temps de corrélation de la perturbation $\mathcal{H}(t)$, dont la valeur exacte sera discutée en détail dans le $\S 2$ suivant. La condition (B.2) exprime que, avant d'avoir eu le temps d'agir de façon importante sur un atome donné, la perturbation à laquelle il est soumis a perdu toute «mémoire" de sa valeur initiale. Dans ce cas, l'évolution de la matrice densité $\rho(t)$ de l'ensemble des atomes sous l'effet du champ inhomogène est donnée par [1] [16] :

$$
\begin{aligned}
& \left(\frac{\mathrm{d} \rho}{\mathrm{d} t}\right)_{\mathrm{rel}}=-\frac{1}{\hbar^{2}} \int_{0}^{\infty} \mathrm{d} \tau \times \\
& \left.\times\left[\overline{\mathscr{H}(t),\left[U_{0}(t, t-\tau) \mathfrak{H}(t-\tau)\right.} U_{0}^{+}(t, t-\tau), \rho(t)\right]\right] .
\end{aligned}
$$

Dans (B.3), la barre qui apparaît au-dessus d'une partie du second membre signifie qu'il faut prendre la valeur moyenne sur l'ensemble des atomes étudiés de l'expression qui y figure ; $U_{0}(t, t-\tau)$ désigne l'opérateur d'évolution libre entre les instants $t$ et $t-\tau$ qui, pour des atomes plongés dans un champ magnétique parallèle à $O z$ d'intensité $-\omega_{0} / \gamma$, s'écrit :

$$
U_{0}(t, t-\tau)=\mathrm{e}^{-i \omega_{0} \sigma_{z} \tau / 2} .
$$

D'autre part, l'expression de la matrice densité $\rho(t)$ en fonction des composantes $M_{x}, M_{y}$ et $M_{z}$ de l'aimantation $\mathbf{M}$ du niveau fondamental est :

$$
\begin{aligned}
\rho(t) & =\frac{1}{2}\left[1+\frac{2}{\gamma \hbar} \boldsymbol{\sigma} \cdot \mathbf{M}\right] \\
& =\frac{1}{2}\left[1+\frac{2}{\gamma \hbar}\left(M_{z} \sigma_{z}+\frac{M_{+} \sigma_{-}+M_{-} \sigma_{+}}{2}\right)\right]
\end{aligned}
$$

(on a posé $M_{ \pm}=M_{x} \pm i M_{y}$ ). En reportant (B.1) et (B.5) dans (B.3), on voit apparaître un certain nombre de doubles commutateurs des opérateurs $\sigma_{+}$, $\sigma_{-}$et $\sigma_{z}$ dont le calcul ne présente pas de difficulté ; et, comme :

$\left\{\begin{array}{l}U_{0}(t, t-\tau) \sigma_{ \pm} U_{0}^{+}(t, t-\tau)=\mathrm{e}^{\mp i \omega_{0} \tau} \sigma_{ \pm} \\ U_{0}(t, t-\tau) \sigma_{z} U_{0}^{+}(t, t-\tau)=\sigma_{z}\end{array}\right.$

on obtient, tous calculs faits :

$$
\left\{\begin{aligned}
\left(\frac{\mathrm{d}}{\mathrm{d} t} M_{+}\right)_{\text {rel }}=-\left[\frac{1}{2} R_{+-}\right. & \left.+R_{z z}\right] M_{+}+ \\
& +R_{z+} M_{z}+\frac{R_{++}}{2} M_{-} \\
\left(\frac{\mathrm{d}}{\mathrm{d} t} M_{z}\right)_{\text {rel }}=\frac{1}{2} R_{-z} M_{+} & -\frac{1}{2}\left[R_{+-}+R_{-+}\right] M_{z} \\
& +\frac{1}{2} R_{+z} M_{-} \\
\left(\frac{\mathrm{d} .7)}{\mathrm{d} t} M_{-}\right)_{\mathrm{rel}}=\frac{R_{--}}{2} M_{+} & +R_{z-} M_{z} \\
& -\left[\frac{1}{2} R_{-+}+R_{z z}\right] M_{-} .
\end{aligned}\right.
$$

Dans ces égalités, les coefficients de relaxation $R$ sont définis par :

$$
\begin{aligned}
& R_{z z}=\int_{0}^{\infty} \mathrm{d} \tau \overline{\Omega_{1 z}[\mathbf{r}(t)] \Omega_{1 z}[\mathbf{r}(t-\tau)]} \times \\
& \times \cos (\omega t) \cos [\omega(t-\tau)] \\
& R_{+z}=\int_{0}^{\infty} \mathrm{d} \tau \overline{\Omega_{1+}[\mathbf{r}(t)] \Omega_{1 z}[\mathbf{r}(t-\tau)]} \times \\
& \times \cos (\omega t) \cos [\omega(t-\tau)] \\
& R_{z+}=\int_{0}^{\infty} \mathrm{d} \tau \mathrm{e}^{i \omega_{0 \tau} \tau} \overline{\Omega_{1 z}[\mathrm{r}(t)] \Omega_{1+}[\mathrm{r}(t-\tau)]} \times \\
& \times \cos (\omega t) \cos [\omega(t-\tau)]
\end{aligned}
$$




$$
\begin{aligned}
& R_{++}=\int_{0}^{\infty} \mathrm{d} \tau \mathrm{e}^{i \omega_{0} \tau} \overline{\Omega_{1+}[\mathbf{r}(t)] \Omega_{1+}[\mathbf{r}(t-\tau)]} \times \\
& \times \cos (\omega t) \cos [\omega(t-\tau)] \\
& R_{-+}=\int_{0}^{\infty} \mathrm{d} \tau \mathrm{e}^{i \omega_{0} \tau} \overline{\Omega_{1-}[\mathbf{r}(t)] \Omega_{1+}[\mathbf{r}(t-\tau)]} \times \\
& \times \cos (\omega t) \cos [\omega(t-\tau)] \\
& R_{-z}=R_{+z}^{*}, \quad R_{z-}=R_{z+}^{*}, \\
& R_{--}=R_{++}^{*}, \quad R_{+-}=R_{-+}^{*}
\end{aligned}
$$

(les coefficients $R$ dont le deuxième indice est + ont $\mathrm{e}^{i \omega_{0} \tau}$ dans l'intégrale sur $\tau$, ceux dont le deuxième indice est - ont $\mathrm{e}^{-i \omega_{0} \tau}$, l'exponentielle étant absente pour ceux dont le deuxième indice est $z$ ).

Nous avons donc calculé la matrice de relaxation $(R)$ qui permet d'écrire l'évolution de $\mathbf{M}$ due au champ de radiofréquence inhomogène sous la forme :

$$
\left(\frac{\mathrm{d}}{\mathrm{d} t} \mathbf{M}\right)_{\mathrm{rel}}=(R) \mathbf{M}
$$

Les éléments de matrice de $(R)$ dans la base $M_{+}$, $M_{z}, M_{-}$apparaissent explicitement dans (B.7). Cependant, pour la suite, il nous sera commode d'utiliser l'expression de $(R)$ dans la base $M_{x}, M_{y}$, $M_{z}$, expression qui est, bien sûr, réelle. Dans $(R)$, les termes indépendants du champ $\mathbf{B}_{0}$ s'expriment en fonction des coefficients réels :

$$
\begin{array}{r}
K_{x_{i}, x_{j}}=\int_{0}^{\infty} \mathrm{d} \tau \overline{\Omega_{1 x_{i}}[\mathrm{r}(t)] \Omega_{1 x_{j}}[\mathrm{r}(t-\tau)]} \times \\
\times \cos (\omega t) \cos [\omega(t-\tau)]
\end{array}
$$

où $x_{i}, x_{j}=x, y$ ou $z$; les $K$, qui dépendent de $\omega$ mais pas de $\omega_{0}$, seront appelés coefficients non résonnants. Pour les coefficients de la relaxation dépendant de $\omega_{0}$, nous introduisons les quantités réelles $C$ et $S$ définies par :

$$
\begin{aligned}
C_{x_{i}, x_{j}}=\int_{0}^{\infty} \mathrm{d} \tau & \cos \omega_{0} \tau \overline{\Omega_{1 x_{i}}[\mathbf{r}(t)] \Omega_{1 x_{j}}[\mathbf{r}(t-\tau)]} \times \\
& \times \cos (\omega t) \cos [\omega(t-\tau)] \quad(\mathbf{B} .10 b)
\end{aligned}
$$

(l'expression de $S$ étant simplement obtenue en remplaçant $\cos \omega_{0} \tau$ par $\left.\sin \omega_{0} \tau\right)$ que nous appellerons «coefficients résonnants" (ils deviennent importants si $\omega_{0} \simeq \omega$ ). On montre aisément que :

$$
\begin{aligned}
& R_{z z}=K_{z z} \\
& R_{+z}=K_{x z}+i K_{y z} \\
& R_{z+}=C_{z x}-S_{z y}+i\left(S_{z x}+C_{z y}\right) \\
& R_{++}=\left(C_{x x}-C_{y y}-S_{x y}-S_{y x}\right)+i\left(C_{x y}+C_{y x}+S_{x x}-S_{y y}\right) \\
& R_{-+}=\left(C_{x x}+C_{y y}-S_{x y}+S_{y x}\right)+i\left(C_{x y}-C_{y x}+S_{x x}+S_{y y}\right)
\end{aligned}
$$

d'où l'on déduit l'expression suivante de la matrice $(R)$, dans la base $O x, O y, O z$ :

$$
(R)=\left(\begin{array}{ccc}
-\left[K_{z z}+C_{y y}+S_{y x}\right] & {\left[-S_{y y}+C_{y x}\right]} & {\left[C_{z x}-S_{z y}\right]} \\
{\left[C_{x y}+S_{x x}\right]} & -\left[C_{x x}-S_{x y}+K_{z z}\right] & {\left[S_{z x}+C_{z y}\right]} \\
{\left[K_{x z}\right]} & {\left[K_{y z}\right]} & -\left[C_{x x}+C_{y y}-S_{x y}+S_{y x}\right]
\end{array}\right) .
$$

Lorsque la condition de rétrécissement par le mouvement est satisfaite, les équations que nous avons obtenues sont générales et donnent l'évolution de l'aimantation $\mathbf{M}$, quelle que soit la loi qui régit le mouvement aléatoire des atomes dans la cellule; cette loi n'interviendra que dans le calcul des valeurs moyennes sur tous les atomes, qui sont symbolisées par des barres horizontales dans l'expression des coefficients $R$. En effet, désignons par $F\left(\mathbf{r}, t ; \mathbf{r}^{\prime}, t^{\prime}\right) d^{3} r$ la probabilité pour que, sachant qu'un atome se trouve en $\mathbf{r}^{\prime}$ à l'instant $t^{\prime}$, il soit à un instant $t$ ultérieur dans un volume $d^{3} r$ centré en $\mathbf{r}$; un raisonnement simple montre alors que :

$$
\begin{array}{r}
\overline{\Omega_{1-}[\mathbf{r}(t)] \Omega_{1+}[\mathbf{r}(t-\tau)]}=\int d^{3} r f(\mathbf{r}, t) \Omega_{1-}(\mathbf{r}) \times \\
\times \int d^{3} r^{\prime} F\left(\mathbf{r}, t ; \mathbf{r}^{\prime}, t-\tau\right) \Omega_{1+}\left(\mathbf{r}^{\prime}\right)
\end{array}
$$

où $f(\mathbf{r}, t)$ est la densité relative $\left(\int f(\mathbf{r}, t) d^{3} r=1\right)$ des atomes à l'instant $t$ (des formules semblables à (B.13) peuvent immédiatement être écrites pour les autres composantes de $\boldsymbol{\Omega}_{\mathbf{1}}(\mathbf{r})$ ). Si nous connaissons. la fonction $F\left(\mathbf{r}, t ; \mathbf{r}^{\prime}, t^{\prime}\right)$, nous pouvons calculer l'intégrale figurant dans (B.13), puis les coefficients $R$ grâce à (B.8), et le problème est en principe résolu. Dans le paragraphe suivant, nous allons effectuer ces calculs dans le cas particulier où le mouvement des atomes est régi par l'équation de diffusion.

B. 2 Application a la Diffusion D'atomes dans UN CHAMP INHOMOGÈNE OSCILLANT. - Pour un ensemble d'atomes diffusant dans une cellule sphérique de rayon $R_{0}$, dont les parois sont supposées parfaitement réfléchissantes (elles ne produisent aucune désorientation des atomes), la fonction $F\left(\mathbf{r}, t ; \mathbf{r}^{\prime}, t^{\prime}\right)$ 
ne dépend que de $\tau=t-t^{\prime}$ et vaut (voir appendice, formule (18)) :

$$
\begin{aligned}
F\left(\mathbf{r}, t ; \mathbf{r}^{\prime}, t^{\prime}\right. & =t-\tau)=F\left(\mathbf{r}, \mathbf{r}^{\prime} ; \tau\right) \\
& =\frac{1}{V}+\sum_{n l m} \varphi_{n, l, m}^{*}\left(\mathbf{r}^{\prime}\right) \varphi_{n, l, m}(\mathbf{r}) \mathrm{e}^{-\tau / \tau_{n, l}}
\end{aligned}
$$

où $V=4 \pi R_{0}^{3} / 3$ est le volume de la cellule, $\varphi_{n, l, m}(\mathbf{r})$ la fonction spatiale associée au mode repéré par les indices $n, l, m$, de temps de diffusion $\tau_{n, l}$. Les expressions précises de $\varphi_{n, l, m}$ et de $\tau_{n, l}$ sont $\left({ }^{8}\right)$ :

$$
\varphi_{n, l, m}(r, \theta, \varphi)=\alpha_{n, l} j_{l}\left(\beta_{n}^{l} r / R_{0}\right) Y_{l}^{m}(\theta, \varphi)
$$

et :

$$
\tau_{n, l}=\frac{1}{D}\left(\frac{R_{0}}{\beta_{n}^{l}}\right)^{2}
$$

$D$ étant le coefficient de diffusion des atomes dans la cellule, $Y_{l}^{m}(\theta, \varphi)$ l'harmonique sphérique d'ordres $l$ et $m, j_{l}$ la fonction de Bessel sphérique d'ordre $l$ et $\beta_{n}^{l}$ le $n$-ième zéro de sa dérivée :

$$
j_{l}^{\prime}\left(\beta_{n}^{l}\right)=0
$$

$\left(\alpha_{n, l}\right.$ est un coefficient de normalisation défini dans l'appendice ; cf. (10)). Compte tenu de (B.13) et en supposant que :

$$
f(\mathbf{r}, t)=\frac{1}{V}
$$

(densité uniforme des atomes), il apparaît dans les coefficients de relaxation $R$ des intégrales de la forme :

$$
\int d^{3} r \varphi_{n, l, m}(\mathbf{r}) \Omega_{1-}(\mathbf{r}) \int d^{3} r^{\prime} \varphi_{n, l, m}^{*}\left(\mathbf{r}^{\prime}\right) \Omega_{1+}\left(\mathbf{r}^{\prime}\right)
$$

qui s'écrivent $\left\langle\Omega_{1+} \mid \varphi_{n, l, m}\right\rangle\left\langle\varphi_{n, l, m} \mid \Omega_{1+}\right\rangle$ en utilisant la notation simplifiée :

$$
\langle\varphi \mid \chi\rangle=\int d^{3} r \varphi^{*}(\mathbf{r}) \chi(\mathbf{r})
$$

(l'intégrale étant étendue à toute la cellule). Nous supposons ici que la valeur moyenne $\left\langle\boldsymbol{\Omega}_{1}(\mathbf{r})\right\rangle$ est nulle (champ de radiofréquence purement inhomogène) ; c'est la raison pour laquelle le terme en $1 / V$ dans (B.14) disparaît lorsqu'on le reporte dans (B. 13) $\left({ }^{9}\right)$.

$\left.{ }^{8}\right) \mathrm{Au}$ lieu des fonctions $\varphi_{n, l, m}(\mathbf{r})$ qui sont complexes (sauf si $m=0$ ), on peut prendre des fonctions réelles $\xi_{n, l, \mid m}^{ \pm}(\mathbf{r})$ proportionnelles à $\varphi_{n, l, m}(\mathbf{r}) \pm \varphi_{n, l,-m}(\mathbf{r})$.

$\left({ }^{9}\right)$ Si le champ de radiofréquence comportait une partie homogène, il ne serait évidemment pas suffisant d'en tenir compte dans le cadre du présent calcul en gardant simplement la contribution du terme en $1 / V$ : la partie homogène du champ agit sur les atomes non pas comme une perturbation aléatoire, mais certaine, et ne donne pas une relaxation de $\mathbf{M}$ (en d'autres termes, pour cette partie homogène, le temps de corrélation serait infini, ce qui exclut qu'elle puisse satisfaire la condition de rétrécissement par le mou-
Les valeurs moyennes sur l'ensemble des atomes ayant ainsi été calculées dans les expressions (B.8) des coefficients $R$, il reste à effectuer l'intégration sur $\tau$ des quantités obtenues, multipliées par $\cos [\omega(t-\tau)]$. Un calcul élémentaire donne alors :

$$
\begin{aligned}
& R_{z z}=\sum_{n, l, m}\left\langle\Omega_{1 z} \mid \varphi_{n, l, m}\right\rangle\left\langle\varphi_{n, l, m} \mid \Omega_{1 z}\right\rangle \times \frac{1}{2 V} \times \\
& \times\left\{\frac{\tau_{n, l}}{1+\left(\omega \tau_{n, l}\right)^{2}}[1+\cos 2 \omega t]+\frac{\omega \tau_{n, l}^{2}}{1+\left(\omega \tau_{n, l}\right)^{2}} \sin 2 \omega t\right\}
\end{aligned}
$$

$R_{+z}$ ayant la même expression, après remplacement de $\left\langle\Omega_{1 z} \mid \varphi_{n, l, m}\right\rangle$ par $\left\langle\Omega_{1-} \mid \varphi_{n, l, m}\right\rangle$. De même, il vient :

$$
\begin{aligned}
& R_{-+}=\sum_{n, l, m}\left\langle\Omega_{1+} \mid \varphi_{n, l, m}\right\rangle\left\langle\varphi_{n, l, m} \mid \Omega_{1+}\right\rangle \times \frac{1}{4 V} \times \\
& \times\left\{\left[\frac{\tau_{n, l}}{1+\left(\omega-\omega_{0}\right)^{2} \tau_{n, l}^{2}}+\mathrm{id}\left(-\omega_{0}\right)\right](1+\cos 2 \omega t)\right. \\
& +\left[\frac{\left(\omega-\omega_{0}\right) \tau_{n, l}^{2}}{1+\left(\omega-\omega_{0}\right)^{2} \tau_{n, l}^{2}}+\mathrm{id}\left(-\omega_{0}\right)\right] \sin 2 \omega t \\
& +i\left[\frac{\tau_{n, l}}{1+\left(\omega-\omega_{0}\right)^{2} \tau_{n, l}^{2}}-\mathrm{id}\left(-\omega_{0}\right)\right] \sin 2 \omega t \\
& \left.+i\left[\frac{\left(\omega+\omega_{0}\right) \tau_{n, l}^{2}}{1+\left(\omega+\omega_{0}\right)^{2} \tau_{n, l}^{2}}-\mathrm{id}\left(-\omega_{0}\right)\right](1+\cos 2 \omega t)\right\}
\end{aligned}
$$

[les notations id $\left(-\omega_{0}\right)$ signifient qu'il faut dans chacun des crochets ajouter, ou retrancher, la même expression où le signe de $\omega_{0}$ a été changé]. Les expressions complètes de $R_{z+}$ et $R_{++}$sont semblables à celle de $R_{-+}$, la seule différence étant que $\left\langle\Omega_{1+} \mid \varphi_{n, l, m}\right\rangle$ est remplacé par $\left\langle\Omega_{1 z} \mid \varphi_{n, l, m}\right\rangle$ dans le premier cas, $\left\langle\Omega_{1-} \mid \varphi_{n, l, m}\right\rangle$ dans le second cas.

Les égalités ainsi obtenues, reportées dans (B.7), permettent donc de connaître exactement l'effet du champ de radiofréquence inhomogène. Nous pouvons d'ailleurs à ce stade préciser la condition de rétrécissement par le mouvement qui fournit une limite de leur validité. La perturbation subie par les atomes possède en fait une infinité de temps de corrélation qui sont les temps de diffusion $\tau_{n, l}$, de sorte que la condition de rétrécissement par le mouvement devient :

$$
\left|\mathbf{\Omega}_{1}(\mathbf{r})\right| \tau_{n, l} \ll 1 \quad \forall n, l ; \quad \forall \mathbf{r} .
$$

Les modes de diffusion de temps $\tau_{n, l}$ les plus longs sont les 3 modes dégénérés $n=l=1, m=1,0,-1$

vement). La façon correcte de tenir compte de la partie homogène du champ de radiofréquence est de la traiter à part de la partie inhomogène, et d'écrire directement sous la forme $\mathrm{d} \mathbf{M} / \mathrm{d} t=\gamma \mathbf{M} \times \mathbf{B}(t)$ l'évolution correspondante de $\mathbf{M}$ (c'est bien ce qui a été fait au $\S \mathrm{A} .2$ où, de plus, ont été discutées les conditions dans lesquelles la partie homogène ne modifie pas la relaxation due à la partie inhomogène). 
(cf. Appendice); par suite, (B.23a) se réduit à :

$$
\left|\mathbf{\Omega}_{1}(\mathbf{r})\right| \tau_{1,1}=\left|\mathbf{\Omega}_{1}(\mathbf{r})\right| \frac{R_{0}^{2}}{D\left(\beta_{1}^{1}\right)^{2}} \ll 1 \quad \forall \mathbf{r}
$$

comme :

$$
\begin{aligned}
\sum_{n, l, m}\left\langle\Omega_{1 z} \mid \varphi_{n, l, m}\right\rangle & \left\langle\varphi_{n, l, m} \mid \Omega_{1 z}\right\rangle \\
& <V \times \operatorname{Max}\left[\Omega_{1 z}(\mathbf{r})\right]^{2}
\end{aligned}
$$

(et des égalités semblables pour les autres composantes de $\boldsymbol{\Omega}_{1}$ ), on voit que tous les coefficients $R$ satisfont :

$$
R \ll 1 / \tau_{1,1} .
$$

L'hypothèse du rétrécissement par le mouvement entraîne donc que les probabilités de relaxation sont faibles devant $1 / \tau_{1,1}$.

B. 3 DisCUSSION : PROPRIÉtÉS DE LA RELAXATION RÉSONNANTE ANISOTROPE PRODUITE PAR UN CHAMP R. F. INHOMOGÈNE. - Dans (B.7), on peut distinguer, parmi les coefficients $R$, deux types de coefficients de couplage : ceux qui donnent des termes diagonaux de la matrice $(R)$, reliant $M_{+}$à $M_{+}, M_{z}$ à $M_{z}$ ou $M_{-}$à $M_{-}$, et ceux qui donnent des termes non diagonaux couplant entre elles des composantes différentes de $\mathbf{M}$. Dans un champ magnétique statique $\mathbf{B}_{0}$, les fréquences propres d'évolution de $M_{+}$, $M_{z}$ et $M_{-}$sont différentes ; les termes non diagonaux sont non séculaires et leur effet est donc négligeable, sauf pour certaines valeurs de $B_{0}$ (résonances) qui seront discutées plus bas $(\S 3 . b . \beta)$. Par contre, les termes diagonaux correspondent toujours (au moins pour leur partie indépendante du temps) à des termes séculaires ; c'est pourquoi nous allons commencer par les étudier.

B.3 a Termes diagonaux de la relaxation. - Considérons tout d'abord le terme de couplage de $M_{z}$ avec lui-même (relaxation longitudinale); le terme diagonal concernant $M_{+}$(relaxation transversale) sera étudié ensuite.

B.3.a. $\alpha$ Relaxation longitudinale. - On voit sur la deuxième égalité (B.7) que le coefficient du terme de couplage de $M_{z}$ avec lui-même est :

$$
\frac{1}{2}\left[R_{+-}+R_{-+}\right]=\operatorname{Re}\left(R_{-+}\right) .
$$

D'après (B.22), ce terme comprend deux parties, l'une constante, l'autre oscillant à la pulsation $2 \omega$. Seule la première de ces deux parties est en fait importante, puisque les termes oscillants sont non séculaires lorsque $\omega \gg R$, condition qui est effectivement satisfaite puisque $\omega \gg 1 / \tau_{1,1}$ [cf. (A.15a) et $1 / \tau_{1,1} \gg R$ [cf. (B.25)]. L'effet du terme diagonal (B.26) est donc de créer une relaxation supplémentaire, caractérisée par un temps $T_{1}^{*}$ tel que :

$$
\begin{aligned}
\frac{1}{T_{1}^{*}}= & \sum_{n, l, m} \frac{1}{4 V}\left|\left\langle\varphi_{n, l, m} \mid \Omega_{1+}\right\rangle\right|^{2} \times \\
& \times\left[\frac{\tau_{n, l}}{1+\left(\omega-\omega_{0}\right)^{2} \tau_{n, l}^{2}}+\mathrm{id}\left(-\omega_{0}\right)\right]
\end{aligned}
$$

avec :

$$
\begin{aligned}
& \left|\left\langle\varphi_{n, l, m} \mid \Omega_{1+}\right\rangle\right|^{2}= \\
& =\int d^{3} r \varphi_{n, l, m}(\mathbf{r}) \Omega_{1-}(\mathbf{r}) \times \int d^{3} r^{\prime} \varphi_{n, l, m}^{*}\left(\mathbf{r}^{\prime}\right) \Omega_{1_{+}}\left(\mathbf{r}^{\prime}\right) .
\end{aligned}
$$

On montre d'ailleurs, en utilisant des modes de diffusion réels [cf. note (8)] rque :

$$
\begin{aligned}
\sum_{m}\left|\left\langle\varphi_{n, l, m} \mid \Omega_{1+}\right\rangle\right|^{2} & =\sum_{m}\left\{\left|\left\langle\varphi_{n, l, m} \mid \Omega_{1 x}\right\rangle\right|^{2}\right. \\
& \left.+\left|\left\langle\varphi_{n, l, m} \mid \Omega_{1 y}\right\rangle\right|^{2}\right\}
\end{aligned}
$$

les composantes sur $O x$ et $O y$ de l'inhomogénéité $\boldsymbol{\Omega}_{1}(\mathbf{r})$ jouent donc un rôle symétrique dans $T_{1}^{*}$.

Les formules (B.27) et (B.28) sont à comparer avec l'expression approchée (A.6) de $1 / T_{1}^{*}$. On remarque tout d'abord que, dans les deux cas, $1 / T_{1}^{*}$ présente un comportement résonnant au voisinage de $\omega_{0}=\omega$; la formule (B.27) prévoit d'ailleurs également une résonance en $\omega_{0}=-\omega$ (terme antirésonnant), ce qui n'est pas étonnant puisque nous avons pris un champ linéaire oscillant ; cependant, si $\omega_{0}$ est positif comme $\omega$, et si $\omega_{0} \gg 1 / \tau_{1,1}$ [cf. (A. 15)], on peut négliger le terme antirésonnant dans le crochet de (B.27). L'expression exacte de $1 / T_{1}^{*}$ ressemble alors nettement à l'expression approchée (A.6). En effet, remplaçons dans (B.27) tous les temps $\tau_{n, l}$ par un seul temps de diffusion $\tau_{d}\left(\tau_{d}\right.$ est par exemple le temps $\tau_{n, l}$ associé aux modes $n, l, m$ dont les poids sont les plus grands) ; il apparaît alors l'expression :

$$
\begin{aligned}
\sum_{n, l, m}\langle & \Omega_{1+}\left|\varphi_{n, l, m}\right\rangle\left\langle\varphi_{n, l, m} \mid \Omega_{1+}\right\rangle= \\
& =\left\langle\Omega_{1+} \mid \Omega_{1+}\right\rangle \\
& =\int d^{3} r\left[\Omega_{1 x}^{2}(\mathbf{r})+\Omega_{1 y}^{2}(\mathbf{r})\right]=V\left\langle\Omega_{1 \perp}^{2}\right\rangle
\end{aligned}
$$

(l'intégrale étant étendue à toute la cellule) et (B.27) se réduit à (A.6). Cependant, l'intérêt de la formule (B.27) est de faire intervenir de façon exacte les différents temps de corrélation de la perturbation (et nous verrons dans la partie expérimentale que plusieurs d'entre eux peuvent être nettement visibles) en donnant leurs poids, qui dépendent de la carte du champ de radiofréquence comme le montre (B.28).

Examinons de façon plus précise comment les variations spatiales de $\Omega_{1+}$ (r) affectent le temps de relaxation $T_{1}^{*}$. La fonction $\Omega_{1+}(\mathbf{r})$ étant, d'après les équations de Maxwell, harmonique $\left({ }^{10}\right)$ :

$$
\Delta \Omega_{1+}(\mathbf{r})=0
$$

elle peut être développée sous la forme :

$$
\Omega_{1+}(\mathbf{r})=\sum_{l \neq 0, m} c_{l, m} r^{l} Y_{l}^{m}(\theta, \varphi)
$$

$\left({ }^{10}\right)$ Nous supposons ici qu'aucun des fils parcourus par des courants qui créent le champ inhomogène ne traverse la cellule. 
[nous excluons ici le terme $l=0$ qui correspondrait à une partie homogène du champ de radiofréquence ; cf. note $\left({ }^{9}\right)$ ]. Il vient alors, d'après (B.15) :

$$
\begin{aligned}
\left\langle\varphi_{n, l, m} \mid \Omega_{1+}\right\rangle= & c_{l, m} \frac{1}{\sqrt{R_{0}^{3} Q_{n, l}}} \times \\
& \times \int_{0}^{R_{0}} j_{l}\left(\beta_{n}^{l} r / R_{0}\right) r^{l} r^{2} \mathrm{~d} r .
\end{aligned}
$$

L'intégrale de normalisation $Q_{n, l}$ est définie dans l'appendice [éq. (11)]. Posons d'autre part :

$$
I_{n, l}=\int_{0}^{1} j_{l}\left(\beta_{n}^{l} \rho\right) \rho^{l+2} \mathrm{~d} \rho .
$$

Pour finir, il vient :

$$
\begin{aligned}
\frac{1}{T_{1}^{*}}=\sum_{n, l \neq 0, m} \mid c_{l, m} & \left.\right|^{2} \frac{3\left|I_{n, l}\right|^{2}}{16 \pi Q_{n, l}} \times \\
& \times R_{0}^{2 l} \frac{\tau_{n, l}}{1+\left(\omega-\omega_{0}\right)^{2} \tau_{n, l}^{2}} .
\end{aligned}
$$

Notons que, si la carte du champ (c'est-à-dire les coefficients $c_{l, m}$ ) est modifiée, on change bien sûr les poids des modes associés à des valeurs différentes de $l$ ou de $m$, mais pas le poids relatif de tous les modes ne différant que par les valeurs de $n$. De plus, les formules (B.34) permettent de savoir comment varie $T_{1}^{*}$ en fonction du rayon $R_{0}$ de la cellule, lorsque les coefficients $c_{l, m}$ sont fixés (c'est-à-dire pour une configuration donnée des fils créant le champ RF inhomogène, et des intensités données les parcourant). A résonance $\left(\omega_{0}=\omega\right)$, la contribution $\left(1 / T_{1}^{*}\right)_{n, l, m}$ de chaque mode $\{n, l, m\}$ à la probabilité de relaxation est telle que :

$$
\left(1 / T_{1}^{*}\right)_{n, l, m} \propto R_{0}^{2(l+1)} .
$$

Par contre, loin de la résonance $\left[\left|\omega-\omega_{0}\right| \tau_{n, l} \gg 1\right]$, on a :

$$
\left(1 / T_{1}^{*}\right)_{n, l, m} \propto R_{0}^{2(l-1)}
$$

Si seul le mode le plus bas $(l=1)$ est pratiquement présent, on a une probabilité de relaxation proportionnelle à $R_{0}^{4}$ à résonance, loin de la résonance une relaxation indépendante de $R_{0}$. Dans le cas opposé où la carte du champ de radiofréquence est suffisamment complexe pour qu'apparaissent des modes de diffusion de $l$ élevés, la probabilité de relaxation varie très vite avec $R_{0}$ [rappelons que toute cette discussion suppose que $R_{0}$ ne dépasse pas le domaine où la condition (B.23b) reste satisfaite].

B.3.a. $\beta$ Relaxation transversale; déplacement de la fréquence de résonance. - On voit sur (B.7) que le terme de couplage de $M_{+}$avec lui-même a pour coefficient :

$$
-\left[\frac{1}{2} R_{+-}+R_{z z}\right]
$$

$R_{z z}$ est un coefficient réel, mais $R_{+-}$est en général complexe; par suite, ce terme correspond non seulement (pour sa partie réelle) à une probabilité de relaxation transversale $1 / T_{2}^{*}$, mais également (pour sa partie imaginaire) à un déplacement de la fréquenee propre d'oscillation de $M_{+}$. Commençons par l'étude de $T_{2}^{*}$; nous avons :

$$
\frac{1}{T_{2}^{*}}=\frac{1}{2 T_{1}^{*}}+R_{z z}^{0}
$$

où $R_{z z}^{0}$ est donné par les termes constants de (B.21) [comme dans le $\S \propto$ précédent, nous négligeons l'effet des termes oscillants dans la relaxation, qui sont non séculaires]. On remarque tout d'abord que seul le terme en $1 / T_{1}^{*}$ a un comportement résonnant lorsque $\omega_{0} \simeq \omega$, alors que $R_{z z}^{0}$ est indépendant de $\omega_{0}$; en fait, $R_{z z}^{0}$ reste négligeable tant que $\omega \gg\left|\omega-\omega_{0}\right|$, $1 / \tau_{n, l}$, et :

$$
\frac{1}{T_{2}^{*}} \simeq \frac{1}{2 T_{1}^{*}}
$$

ce qui signifie que la relaxation transversale est deux fois plus lente que la relaxation longitudinale. Physiquement, ceci peut se comprendre par un passage dans le référentiel tournant, où seules les deux composantes transversales du champ de radiofréquence deviennent statiques, la composante longitudinale restant oscillante (ce qui permet de négliger son effet) ; les deux composantes statiques contribuent alors à la relaxation de $M_{z}$, alors qu'une seule d'entre elles peut contribuer à la relaxation de la composante $M_{X}$ par exemple ( $O X$ étant un axe du référentiel tournant perpendiculaire à $O Z$ ) : la composante $\Omega_{1 Y}$ sur l'axe $O Y$ perpendiculaire à $O X$. Par suite, il n'est pas étonnant que $M_{z}$ relaxe deux fois plus vite que $M_{X}$.

Le terme en $R_{z z}^{0}$ correspond à ce que l'on nomme souvent le terme adiabatique dans la relaxation de la cohérence du système. Pour un champ inhomogène statique, ce terme adiabatique serait de l'ordre de $\left\langle\Omega_{1 \perp}^{2}\right\rangle \tau_{\mathrm{d}}$ [comme le montre (B.21) lorsqu'on y porte $\omega=0]$, tandis que $1 / T_{1}^{*}$ serait de l'ordre de $\left\langle\Omega_{1 \perp}^{2}\right\rangle \tau_{\mathrm{d}} /\left[1+\left(\omega_{0} \tau_{\mathrm{d}}\right)^{2}\right]$; pour un champ inhomogène statique, le terme adiabatique est donc presque toujours le terme dominant dans la relaxation transversale (ce n'est qu'en champ très faible, lorsque $\omega_{0} \tau_{\mathrm{d}} \lesssim 1$, que le terme en $1 / T_{1}^{*}$ devient comparable au terme adiabatique). Par contre, pour un champ de radiofréquence inhomogène comme celui étudié ici, il en va autrement : le fait que la composante $\Omega_{1 z}$ soit modulée réduit le terme adiabatique dans un facteur $\left(1 / \omega \tau_{\mathrm{d}}\right)^{2}$, alors que $1 / T_{1}^{*}$ prend toujours une valeur maximale de l'ordre de $\left\langle\Omega_{1 \perp}^{2}\right\rangle \tau_{\mathrm{d}}$; par suite, c'est le terme adiabatique qui est généralement négligeable (sauf lorsque $\omega_{0} \gg \omega$ cas auquel, comme 
pour un champ inhomogène statique, le terme adiabatique redevient dominant).

La partie imaginaire de $R_{+-}$donne un déplacement $\delta \omega_{0}^{*} / 2 \pi$ de la fréquence d'évolution de $M_{+}$:

$$
\begin{aligned}
\delta \omega_{0}^{*} & =-\frac{1}{2} \operatorname{Im}\left\{R_{+-}\right\} \\
& =\frac{1}{2} \operatorname{Im}\left\{R_{-+}\right\} .
\end{aligned}
$$

D'après (B.22), on a (comme plus haut, nous ne gardons dans $R$ que la partie constante des termes résonnants pour $\omega_{0} \simeq \omega$ ):

$$
\begin{aligned}
\delta \omega_{0}^{*}=\frac{1}{2} \sum_{n, l \neq 0, m} \frac{\left|\left\langle\varphi_{n, l, m} \mid \Omega_{1}\right\rangle\right|^{2}}{4 V} \times \\
\quad \times \frac{\left(\omega_{0}-\omega\right) \tau_{n, l}^{2}}{1+\left(\omega_{0}-\omega\right)^{2} \tau_{n, l}^{2}} .
\end{aligned}
$$

Dans l'approximation où tous les temps de diffusion sont remplacés par un temps unique $\tau_{\mathrm{d}}$, cette expression devient simplement :

$$
\begin{aligned}
\delta \omega_{0}^{*} & \simeq \frac{1}{2} \frac{\left(\left\langle\Omega_{1 \perp}^{2}\right\rangle / 4\right)\left(\omega_{0}-\omega\right) \tau_{\mathrm{d}}^{2}}{1+\left(\omega_{0}-\omega\right)^{2} \tau_{\mathrm{d}}^{2}} \\
& \simeq\left(\omega_{0}-\omega\right) \tau_{\mathrm{d}} \times \frac{1}{2 T_{1}^{*}}
\end{aligned}
$$

La relaxation produite par le champ de radiofréquence inhomogène entraîne donc l'existence d'un déplacement de la fréquence de résonance de $\mathbf{M}$. Ce déplacement, nul pour $\omega_{0}=\omega$, peut devenir notable lorsque $\omega_{0} \neq \omega$; dès que $\left|\omega_{0}-\omega\right| \tau_{\mathrm{d}} \gg 1$, il est en fait beaucoup plus important que l'élargissement de la résonance lié au raccourcissement de $T_{2}^{*}$; c'est pourquoi il est aisément observable expérimentalement (ce qui n'est pas le cas lorsque l'inhomogénéité est statique, l'élargissement de la résonance provenant du terme adiabatique de la relaxation transversale étant toujours comparable, ou supérieur, au déplacement).

Contrairement à la probabilité de relaxation $1 / T_{1}^{*}$, qui est due aux transitions réelles subies par les atomes sous l'effet du champ magnétique aléatoire, le déplacement $\delta \omega_{0}^{*}$ provient des transitions virtuelles induites par ce même champ [16] : ces transitions virtuelles font apparaître un couplage entre les deux niveaux non perturbés des spins, d'énergies $\pm \hbar \omega_{0} / 2$, couplage qui change leurs énergies.

Remarques. - (i) Nous avons négligé le terme antirésonnant dans $\operatorname{Im}\left\{R_{-+}\right\}$pour obtenir $\delta \omega_{0}^{*}$. D'après (B.22), lorsque $\omega_{0} \simeq \omega$ et $\omega \gg 1 / \tau_{n, l}$, ce terme vaut :

$$
\begin{aligned}
\delta \omega_{0}^{* \prime} & \simeq \frac{1}{2 \omega_{0}} \sum_{n, l \neq 0, m} \frac{\left|\left\langle\varphi_{n, l, m} \mid \Omega_{1+}\right\rangle\right|^{2}}{4 V} \\
& =\frac{1}{2 \omega_{0}} \frac{\left\langle\Omega_{1 \perp}^{2}\right\rangle}{4}
\end{aligned}
$$

$\delta \omega_{0}^{* \prime}$ est analogue au déplacement de Bloch-Siegert [18] qui, pour un ensemble de spins soumis à l'action d'un champ linéaire homogène oscillant d'intensité $-\omega_{1} / \gamma$, vaut $\omega_{1}^{2} / 4 \omega_{0}$. Cependant la formule (B.40) montre que, pour un ensemble de spins se déplaçant rapidement dans un champ oscillant inhomogène, $\delta \omega_{0}^{* \prime}$ n'est pas simplement la moyenne sur la cellule des déplacements de Bloch-Siegert en chaque point $\mathbf{r}$, mais sa moitié seulement.

ii) L'expression (B.14) de la fonction $F$ que nous avons prise n'est certainement pas valable pour les temps $\tau$ très courts. Cette expression ayant été obtenue à partir de l'équation de diffusion, elle suppose implicitement que $\tau$ est grand devant le temps $\Delta \tau_{\mathrm{c}}$ qui sépare deux collisions cinétiques successives subies par un atome donné (par exemple, pour un gaz d'hélium à 1 torr, $\Delta \tau_{\mathrm{c}} \simeq 10^{-7} \mathrm{~s}$ [4]). En effet, le mouvement très rapide des atomes entre deux collisions n'obéit évidemment pas à l'équation de diffusion : ce n'est que sur des temps longs $\left(\tau \gg \Delta \tau_{\mathrm{c}}\right)$ que ces mouvements rapides se moyennent pour donner un processus de diffusion. Tant que les intégrales sur $\tau$ qui figurent dans les coefficients $R$ sont peu sensibles aux valeurs de $F$ pour $\tau \lesssim \Delta \tau_{\text {c }}$, les calculs que nous avons développés jusqu'ici sont valables ; c'est effectivement le cas lorsque $\omega_{0} \Delta \tau_{\mathrm{c}} \ll 1$. Par contre, si $\omega_{0} \gtrsim\left(\Delta \tau_{c}\right)^{-1}$, la relaxation devient sensible au mouvement rapide des atomes et ses caractéristiques peuvent être très différentes $\left(\Delta \tau_{\mathrm{c}}\right.$ joue le rôle d'un temps de corrélation court de la perturbation). C'est ce qui explique pourquoi les références [4] et [5] donnent des expressions de $T_{1}^{*}$, valables en champ très fort seulement, très différentes des nôtres (elles dépendent de $\Delta \tau_{\mathrm{c}}$, mais pas de $R_{0}$ ). Bien sûr, les deux théories doivent donner le même résultat dans le domaine intermédiaire

$$
\left(\tau_{1,1}\right)^{-1} \ll \omega_{0} \ll\left(\Delta \tau_{\mathrm{c}}\right)^{-1} ;
$$

'c'est bien ce que l'on constate, du moins si on ne retient de l'expression (B.34) de $1 / T_{1}^{*}$ que la contribution du mode $l=1$ (dont nous avons effectivement noté plus haut qu'elle est indépendante de $R_{0}$ en champ fort).

B.3.b Termes non diagonaux. - Comme le montre (B.7), l'effet de la relaxation produite par le champ de radiofréquence inhomogène n'est pas simplement de modifier les caractéristiques de l'évolution propre de chacune des composantes $M_{+}$, $M_{z}$ et $M_{-}$prise isolément ; la relaxation introduit également un couplage entre les différentes composantes de M. Jusqu'ici, nous n'avons étudié que les éléments diagonaux de la matrice $(R)$ qui, d'après (B.9), caractérise l'effet de la relaxation sur M. Mais, nous le verrons plus bas, les éléments non diagonaux de $(R)$ peuvent avoir un effet important sur la polarisation $\mathbf{M}$ lorsque le champ $\mathbf{B}_{0}$ passe par certaines valeurs $\left(\omega_{0}=0, \omega\right.$ ou $\left.2 \omega\right)$. C'est pourquoi nous allons étudier de façon plus détaillée la matrice $(R)$, ce qui nous permettra de mieux dégager les propriétés angulaires de la relaxation. 
B.3.b. $\alpha$ Parties scalaire, vectorielle et tensorielle de la relaxation. - La matrice $(R)$ peut se décomposer en trois matrices

$$
(R)=(R)_{\mathrm{S}}+(R)_{\mathrm{V}}+(R)_{\mathrm{T}}
$$

où $(R)_{\mathrm{S}}$ est proportionnelle à la matrice unité (partie scalaire), $(R)_{\mathrm{V}}$ est antisymétrique (partie vectorielle) et $(R)_{\mathrm{T}}$ symétrique à trace nulle (partie tensorielle). Les expressions de ces matrices en fonction des coefficients $K, C, S$ définis en (B.10) sont :

$$
\begin{aligned}
& (R)_{\mathrm{S}}=-\frac{2}{3}\left[C_{x x}+C_{y y}+K_{z z}+S_{y x}-S_{x y}\right] \\
& (R)_{\mathrm{V}}=\left[\begin{array}{ccc}
0 & -V_{z} & V_{y} \\
V_{z} & 0 & -V_{x} \\
-V_{y} & V_{x} & 0
\end{array}\right]
\end{aligned}
$$

avec :

$$
\left\{\begin{array}{l}
V_{x}=\frac{1}{2}\left[K_{y z}-C_{z y}-S_{z x}\right] \\
V_{y}=\frac{1}{2}\left[-K_{x z}+C_{z x}-S_{z y}\right] \\
V_{z}=\frac{1}{2}\left[C_{x y}-C_{y x}+S_{x x}+S_{y y}\right]
\end{array}\right.
$$

et enfin :

$$
(R)_{\mathrm{T}}=\left[\begin{array}{lll}
T_{x x} & T_{x y} & T_{x z} \\
T_{x y} & T_{y y} & T_{y z} \\
T_{x z} & T_{y z} & T_{z z}
\end{array}\right]
$$

On a posé :

$$
\left\{\begin{array}{l}
T_{x x}=\frac{2}{3} C_{x x}-\frac{1}{3}\left[K_{z z}+C_{y y}+S_{y x}+2 S_{x y}\right] \\
T_{y y}=\frac{2}{3} C_{y y}-\frac{1}{3}\left[K_{z z}+C_{x x}-S_{x y}-2 S_{y x}\right] \\
T_{z z}=-T_{x x}-T_{y y} \\
T_{x y}=\frac{1}{2}\left[C_{x y}+C_{y x}\right]+\frac{1}{2}\left[S_{x x}-S_{y y}\right] \\
T_{x z}=\frac{1}{2}\left[C_{z x}+K_{x z}\right]-\frac{1}{2} S_{z y} \\
T_{y z}=\frac{1}{2}\left[C_{z y}+K_{y z}\right]+\frac{1}{2} S_{z x} .
\end{array}\right.
$$

La partie scalaire de la relaxation correspond à une relaxation isotrope de l'aimantation $\mathbf{M}$. A cette relaxation isotrope s'ajoute la relaxation anisotrope liée à la partie tensorielle [la matrice $(R)_{\mathrm{T}}$, symétrique et réelle, a des valeurs propres réelles et correspond donc effectivement à une relaxation pour $\mathbf{M}]$; la combinaison de $(R)_{\mathrm{S}}$ et $(R)_{\mathrm{T}}$ donne donc une relaxation anisotrope, admettant trois axes propres de relaxation [les directions propres de $(R)_{\mathrm{T}}$ ] auxquels sont associées trois constantes de relaxation en général différentes. La symétrie de la relaxation ainsi obtenue est celle d'un ellipsoïde dont les trois axes sont les directions propres de $(R)_{\mathrm{T}}$.

Quant à la partie vectorielle $(R)_{\mathrm{V}}$, elle donne une évolution de $\mathbf{M}$ sous la forme d'un produit vectoriel :

$$
\left(\frac{\mathrm{d} \mathbf{M}}{\mathrm{d} t}\right)_{\mathrm{v}}=\mathbf{V} \times \mathbf{M} .
$$

Cette partie vectorielle ne donne donc pas une relaxation de $\mathbf{M}$, mais une précession de $\mathbf{M}$ autour de $\mathbf{V}$; la matrice $(R)_{\mathrm{V}}$ associe donc à la relaxation magnétique un champ magnétique fictif $\delta \mathbf{B}_{0}^{*}=-\mathbf{V} / \gamma$. La composante sur $O z$ de ce champ $\delta \mathbf{B}_{0}^{*}$, qui vient s'ajouter à $\mathbf{B}_{0}$, correspond évidemment au déplacement $\delta \omega_{0}^{*}$ calculé plus haut.

Remarques. - (i) Lorsque le mouvement des atomes obéit à l'équation de diffusion, la fonction de corrélation $F$ qui permet de calculer les valeurs moyennes dans les coefficients $K, C$ et $S$ est symétrique en $\mathbf{r}$ et $\mathbf{r}^{\prime}$ [on peut s'en assurer aisément en prenant dans (B.14) des fonctions de base réelles au lieu des fonctions (B.15) ; cf. note (8)]. Comme de plus la fonction $f(\mathbf{r}, t)$ dans (B.13) est prise égale à $1 / V$, on constate que $\Omega_{1 x_{i}}[\mathrm{r}(t)] \Omega_{1 x_{j}}[\mathrm{r}(t-\tau)]$ est une fonction symétrique de $x_{i}$ et $x_{j}$. Par suite, il en est de même des coefficients $K, C$ et $S$, ce qui permet de simplifier les égalités (B.43b) et (B.44b). Par exemple, on a $V_{z}=\frac{1}{2}\left[S_{x x}+S_{y y}\right]$.

(ii) Les formules (B.42) et suivantes permettent de savoir comment les symétries de relaxation dépendent de la carte du champ de radiofréquence inhomogène. Supposons par exemple que ce champ soit en tous points perpendiculaire à $O z$ (situation qui peut expérimentalement être réalisée en créant ce champ au moyen de deux longs fils parallèles à $O z$ parcourus par un courant) ; on voit alors immédiatement que le champ magnétique fictif est parallèle à $O z\left(V_{x}=V_{y}=0\right)$, et que l'ellipsoïde de relaxation admet $O z$ comme axe principal $\left(T_{x z}=T_{y z}=0\right):$ la direction $O z$ est direction propre de $(R)_{\mathrm{V}}$ et $(R)_{\mathrm{T}}$ à la fois.

B.3.b. $\beta$ Termes résonnants et non résonnants. Evaluons de façon plus précise les valeurs des coefficients $K, C$ et $S$. Un calcul analogue à celui qui a donné les égalités (B.21) et (B.22) conduit à :

$$
\begin{aligned}
& K_{x_{i} x_{j}}=\sum_{n, l, m}\left\langle\Omega_{1 x_{i}} \mid \varphi_{n, l, m}\right\rangle\left\langle\varphi_{n, l, m} \mid \Omega_{1 x_{j}}\right\rangle \frac{1}{4 V} \times \\
& \times\left\{\frac{2 \tau_{n, l}}{1+\left(\omega \tau_{n, l}\right)^{2}}[1+\cos 2 \omega t]+\frac{2 \omega \tau_{n, l}^{2}}{1+\left(\omega \tau_{n, l}\right)^{2}} \sin 2 \omega t\right\} .
\end{aligned}
$$

Pour $C_{x_{i} x_{j}}$, il faut remplacer l'accolade par :

$$
\begin{aligned}
& \left\{\left[\frac{\tau_{n, l}}{1+\left(\omega-\omega_{0}\right)^{2} \tau_{n, l}^{2}}+\mathrm{id} .\left(-\omega_{0}\right)\right][1+\cos 2 \omega t]+\right. \\
& \left.+\left[\frac{\left(\omega-\omega_{0}\right) \tau_{n, l}^{2}}{1+\left(\omega-\omega_{0}\right)^{2} \tau_{n, l}^{2}}+\mathrm{id}\left(-\omega_{0}\right)\right] \sin 2 \omega t\right\}
\end{aligned}
$$

et, pour $S_{x_{i} x_{j}}$, par :

$$
\begin{aligned}
& \left\{\left[\frac{\tau_{n, l}}{1+\left(\omega-\omega_{0}\right)^{2} \tau_{n, l}^{2}}-\mathrm{id}\left(-\omega_{0}\right)\right] \sin 2 \omega t+\right. \\
& \left.+\left[\frac{\left(\omega+\omega_{0}\right) \tau_{n, l}^{2}}{1+\left(\omega+\omega_{0}\right)^{2} \tau_{n, l}^{2}}-\mathrm{id}\left(-\omega_{0}\right)\right][1+\cos 2 \omega t]\right\} .
\end{aligned}
$$


Nous retrouvons sur ces formules que les coefficients $K$ et $C$ sont égaux lorsque $\omega_{0}=0$, les $S$ étant alors nuls. Lorsque $\omega_{0} \simeq \omega$, les coefficients $C$ et $S$ subissent des variations résonnantes et deviennent beaucoup plus importants (si $\left|\omega-\omega_{0}\right| \ll \omega$ ) que les $K$, qui restent constants; dans ce cas, l'égalité (B.12) montre que l'évolution de $M_{z}$ due à la relaxation ne dépend pratiquement que de $M_{z}$ et non de $M_{x}$ et de $M_{y}\left({ }^{11}\right)$. Physiquement, le rôle privilégié joué par l'axe $O z$ tient au fait que la composante sur $O z$ du champ inhomogène oscillant n'est jamais résonnante (elle reste oscillante dans le référentiel tournant), contrairement aux composantes transversales (dont une partie devient constante dans le référentiel tournant).

B.3.b. $\gamma$ Termes séculaires et non séculaires. Indépendamment du fait qu'ils soient résonnants ou non, les termes de couplage introduits par la relaxation auront un effet important ou au contraire négligeable sur l'aimantation $\mathbf{M}$ suivant qu'ils sont séculaires ou non séculaires $\left({ }^{12}\right)$. En effet, si l'influence d'un terme' non résonnant peut éventuellement se faire sentir lorsque $\omega_{0}$ est assez différent de $\omega$ (les temps de diffusion $\tau_{n, l}$ étant petits, les variations résonnantes des coefficients $C$ et $S$ se font sur des largeurs importantes), l'influence d'un terme non séculaire devient très vite complètement négligeable [l'influence d'un terme de couplage non diagonal se fait sentir dans un domaine dont la largeur est faible, de l'ordre de l'inverse des temps de relaxation du système, beaucoup plus grands que les $\tau_{n, l}$; $\mathrm{cf}$. (B. 25)].

Pour examiner quels termes de la relaxation peuvent devenir séculaires, il faut tenir compte de l'évolution dans le temps des différents éléments de matrice de $(R)$. On voit sur les formules (B.46), (B.47) et (B.48) que les coefficients $K, C$ et $S$ comprennent tous une partie constante et une autre modulée à la pulsation $2 \omega$.

Pour les parties constantes, elles sont toujours séculaires lorsqu'elles correspondent à des termes diagonaux de la relaxation (dans la base $M_{+}, M_{z}, M_{-}$). Nous avons déjà étudié plus haut en détail leur contribution : $(R)_{\mathrm{S}}$ et $(R)_{\mathrm{T}}$ donnent des temps de relaxation $T_{1}^{*}$ et $T_{2}^{*},(R)_{\mathrm{V}}$ le déplacement de fréquence $\delta \omega_{0}^{*}$. En ce qui concerne les termes constants non diagonaux (couplages entre $M_{ \pm}$et $M_{z}$ ou $M_{+}$ et $M_{-}$), ils ne sont séculaires qu'en champ $B_{0}$ nul; $(R)_{\mathrm{v}}$ étant alors également nulle, les effets résonnants ne peuvent alors provenir que de la partie tensorielle $(R)_{\mathrm{T}}$ de la relaxation.

Pour les parties modulées à $2 \omega$, elles peuvent

$\left({ }^{11}\right)$ La matrice $(R)$ n'étant pas hermitique, ceci n'implique pas nécessairement que $O z$ soit direction propre de $(R)$.

$\left({ }^{12}\right)$ Rappelons que, de façon générale, un terme de couplage modulé à la pulsation $\omega_{c}$ est dit séculaire s'il couple deux grandeurs physiques de pulsations propres $\omega_{a}$ et $\omega_{b}$ telles que $\omega_{a}+\omega_{c}=\omega_{b}$. devenir séculaires lorsque $\omega_{0} \simeq \omega$ (termes de couplage entre $M_{+}$et $M_{-}$) ou lorsque $\omega_{0} \simeq 2 \omega$ (termes de couplage entre $M_{ \pm}$et $M_{z}$ ). Lorsque $\omega_{0} \simeq \omega$, seule la partie tensorielle de la relaxation peut coupler $M_{+}$et $M_{-}$et aucun effet n'est à attendre du champ magnétique fictif (ce qui n'est pas étonnant, puisqu'il oscille à $2 \omega$ et non à $\omega$ ). Par contre, lorsque $\omega_{0} \simeq 2 \omega$, des effets résonnants peuvent être attendus à la fois de la relaxation tensorielle et du champ magnétique fictif.

Parmi les différentes possibilités envisagées, discutons plus en détail le cas où $\omega_{0} \simeq \omega$, qui est le plus intéressant puisque certains termes non diagonaux de la relaxation sont alors à la fois résonnants et séculaires : il faut attendre dans ce cas des effets particulièrement marqués du caractère anisotrope de la relaxation. On peut même se demander si l'étude du $\S \mathrm{A}$ est valable puisque, pour calculer la valeur stationnaire de l'orientation longitudinale, nous n'avons tenu compte que du terme diagonal de la relaxation caractérisé par le temps $T_{1}^{*}$; n'y a-t-il pas, lorsque $\omega_{0} \simeq \omega$, des effets résonnants de la relaxation liés aux termes non diagonaux de sa partie tensorielle et, par suite, des modifications profondes de la forme des courbes de résonance? En fait, au moins dans les expériences envisagées jusqu'ici, il n'en est rien. Ceci tient à la symétrie de la relaxation qui, comme nous l'avons signalé plus haut, ne couple la composante $M_{z}$ qu'à elle-même lorsque $\omega_{0} \simeq \omega$. Tant que l'on ne s'intéresse qu'à la composante longitudinale de la polarisation des atomes, il est donc correct de caractériser l'effet de la relaxation due au champ de radiofréquence inhomogène par le seul temps $T_{1}^{*}$. Pour voir dans ce cas des effets résonnants dus à la partie inhomogène du champ de radiofréquence, il faudrait créer et détecter des composantes transversales de l'orientation, situation que nous n'envisagerons pas ici.

B.3.b. $\delta$ Approximation du référentiel tournant. - Dans le modèle simple utilisé au début de cet article ( $(A)$, nous avons raisonné, non dans le référentiel du laboratoire où nous avons établi les équations (B.7), mais directement dans le référentiel tournant, en ignorant délibérément les composantes du champ de radiofréquence qui ne deviennent pas statiques dans ce dernier référentiel. Cette façon de faire conduit à des simplifications (elle fait notamment disparaître la composante longitudinale de l'inhomogénéité) mais, bien sûr, elle n'est qu'une approximation, dont nous allons discuter ici les limites.

Remarquons tout d'abord que les égalités (B.7) et suivantes peuvent être interprétées de deux façons distinctes : ou bien, comme nous l'avons fait jusqu'ici, on considère qu'elles donnent l'évolution de $\mathbf{M}$ dans le référentiel du laboratoire, ou bien on les interprète comme valables dans le référentiel tournant $O X Y Z$; dans ce second cas on effectue, dans les formules (B.8) 
et suivantes, les substitutions :

$$
\begin{aligned}
& \left\{\begin{array}{l}
\omega \rightarrow 0 \\
\omega_{0} \rightarrow \Delta_{0} \\
\Omega_{1 x} \rightarrow \Omega_{1 X}=\Omega_{1 x} / 2 \\
\Omega_{1 y} \rightarrow \Omega_{1 Y}=\Omega_{1 y} / 2
\end{array}\right. \\
& \Omega_{1 z} \rightarrow \Omega_{1 Z}=0 .
\end{aligned}
$$

On obtient alors pour $(R)$ une matrice constante (dans le référentiel tournant) et, du fait de la nullité de $\Omega_{1 Z}$, nettement plus simple que celle qui décrit la relaxation dans le point de vue du laboratoire. Quels sont les termes " oubliés » dans cette simplification? L'examen des formules (B.7) et (B.8) ainsi que des substitutions (B.49) montre que ce sont les termes, soit non résonnants, soit non séculaires lorsque $\omega_{0} \simeq \omega$. En effet, les coefficients $R_{z z}, R_{+z}$ et $R_{-z}$, qui sont non résonnants, font tous intervenir la composante longitudinale de l'inhomogénéité, de sorte qu'ils correspondent donc bien à des coefficients nuls dans l'approximation du référentiel tournant; pour la même raison $R_{z_{+}}$et $R_{z_{-}}$, qui comprennent une partie constante et une autre oscillant à $2 \omega$ ne donnant que des couplages non séculaires entre $M_{z}$ et $M_{ \pm}$, sont nuls dans le référentiel tournant. D'autre part, on vérifie que les parties résonnantes constantes de $R_{-+}$et $R_{+-}$(de même que les parties résonnantes de $R_{++}$et $R_{--}$variant comme $\mathrm{e}^{2 i \omega t}$ et $\mathrm{e}^{-2 i \omega t}$ respectivement) ont bien la même valeur que les coefficients qui leur correspondent dans le référentiel tournant par les substitutions (B.49).

Raisonner directement dans le référentiel tournant revient donc à ne garder que les termes de couplage introduits par la relaxation qui sont à la fois résonnants et séculaires (lorsque $\omega_{0} \simeq \omega$ ). Par exemple, le fait que $\Omega_{1 Z}=0$ permet de voir directement sur (B.12) que l'évolution de $M_{Z}$ n'est pas couplée à $M_{X}$ ou $M_{Y}$ dans ce cas. De même, les égalités (B.43) montrent que, dans le référentiel tournant, $V_{X}$ et $V_{Y}$ sont nuls : nous avons déjà noté plus haut que, si $\omega_{0} \simeq \omega$, seule la composante longitudinale de $\mathbf{V}$ peut être séculaire (on retrouverait de même que cette partie séculaire s'annule lorsqu'on se place exactement à résonance $\omega_{0}=\omega$ ).

Ce type de raisonnement permet également de comprendre dans quelles conditions les termes non diagonaux de la relaxation du champ de radiofréquence (complètement inhomogène) peuvent produire des effets résonnants au voisinage de $\omega_{0}=\omega$ : pour que la composante $M_{z}$ soit couplée à $M_{x}$ et $M_{y}$, il faut que l'inhomogénéité magnétique ait, dans le référentiel tournant, une composante longitudinale; il faut donc que les atomes soient également soumis à l'action d'un champ inhomogène statique dans le référentiel du laboratoire. On peut alors s'attendre (et nous verrons que c'est effectivement le cas) à d'importantes modifications des formes de courbe de résonance au voisinage de $\omega_{0}=\omega$. Il s'agit là en fait d'une situation en pratique courante puisque, dans la plupart des expériences, les atomes étudiés sont soumis à des inhomogénéités magnétiques statiques; nous allons donc étudier ce qui se produit dans ce cas.

B.3.c Cas où le champ statique est également inhomogène. - Dans le référentiel du laboratoire, quelles sont les modifications de la matrice $(R)$ lorsque les inhomogénéités de champ comprennent une partie statique en plus d'une partie modulée ? Tout d'abord, à la matrice de relaxation $(R)$ associée au champ oscillant, il faudra évidemment ajouter la matrice de relaxation du champ statique inhomogène, indépendante du temps (que l'on peut d'ailleurs facilement calculer en faisant $\omega=0$ dans les formules obtenues plus haut); sauf si $\omega_{0}=0$ (champ nul), seuls les termes diagonaux (dans la base $M_{+}, M_{z}, M_{-}$) de cette relaxation statique seront séculaires, et son seul effet sera de modifier $T_{1}$ et $T_{2}$ et éventuellement de produire un déplacement de la fréquence de résonance (mais nous avons vu plus haut que les déplacements de fréquence dus à un champ inhomogène statique sont généralement faibles devant les élargissements correspondants, du fait de l'importance du terme adiabatique) ; on peut par exemple supposer que les effets du champ statique sur les relaxations longitudinale et transversale sont déjà incorporés dans les temps $T_{1}^{0}$ et $T_{2}^{0}$.

Toutefois, il ne suffit pas d'ajouter simplement les effets des champs inhomogènes statique et oscillant : les éléments de matrice de $(R)$ étant des fonctions quadratiques des champs, il existe également des termes croisés, oscillant à la pulsation $\omega$; comme nous allons le voir, ce sont eux qui donnent les effets les plus intéressants. Pour simplifier, nous allons nous limiter ici au cas où $\omega_{0} \simeq \omega$ (de façon plus précise où $\left.\left|\omega_{0}-\omega\right| \tau_{n, l} \ll 1\right)$ et nous n'allons tenir compte que des termes croisés qui sont à la fois résonnants et séculaires. Dans ce but, nous raisonnerons directement dans le référentiel tournant, en ne gardant comme plus haut que les composantes de l'inhomogénéité qui deviennent statiques dans ce référentiel : composante transversale du champ de radiofréquence inhomogène et composante longitudinale du champ inhomogène statique. Le raisonnement est alors exactement le même que dans le paragraphe $b . \delta$ précédent, la seule différence étant que $\Omega_{1 z}$ ne doit plus être pris nul; il faut remplacer (B.49b) par :

$$
\Omega_{1 z} \rightarrow \Omega_{1 Z}=\Omega_{1 z}^{\mathrm{s}}
$$

où $\Omega_{1 z}^{\mathrm{s}}$ est la composante sur $O z$ de l'inhomogénéité statique. Par suite, le coefficient $R_{Z Z}$ est maintenant différent de zéro, ainsi que les 4 coefficients $R_{Z \pm}, R_{ \pm Z}$. On voit sur (B.11) que la présence du coefficient $R_{\mathrm{ZZ}}$, qui est le seul terme à varier quadratiquement en fonction du champ statique inhomogène, se traduit simplement pour la relaxation par un élément diagonal donnant un raccourcissement du temps de relaxation $T_{2}\left(R_{z z}\right.$ est le terme "adiabatique» dû au 
champ inhomogène statique). Ce sont donc effectivement les termes $R_{Z \pm}$ et $R_{ \pm Z}$ qui sont les termes croisés (linéaires à la fois par rapport aux inhomogénéités statique et oscillante) les plus intéressants, puisqu'ils modifient la symétrie de la relaxation; on remarque notamment que $M_{Z}$ est maintenant couplé à $M_{+}$et $M_{-}$. Pour examiner la symétrie de la relaxation dans ce cas, on peut utiliser les formules (B.41) et suivantes [toujours en y effectuant les substitutions (B.49a et $c$ ] ; comme $\left|\Delta_{0}\right| \ll 1 / \tau_{n, l}$, les coefficients $S$ sont nuls dans ces formules et les coefficients $C$ égaux aux coefficients $K$ correspondants. On remarque alors que $\mathbf{V}=0$ : seuls les termes de la relaxation tensorielle anisotrope peuvent donc jouer un rôle dans ce cas ; nous étudierons ce qu'il en est dans le $\S \mathrm{C} .2$ suivant $\left({ }^{13}\right)$.

C. Forme des courbes de résonance. - Maintenant que les diverses caractéristiques de la relaxation sont mieux précisées, nous pouvons reprendre de façon plus détaillée l'étude du $\S \mathrm{A}$ concernant la forme des courbes de résonance, pour savoir dans quelle mesure les prévisions du modèle simple utilisé alors sont correctes. Nous allons voir qu'à quelques modifications près, dues à la présence de plusieurs modes de diffusion, les conclusions obtenues plus haut concernant la courbe large restent effectivement les mêmes; ceci tient au fait que l'existence de la courbe large est liée aux variations résonnantes des termes diagonaux de la relaxation. Par contre, pour la courbe fine, les termes non diagonaux, et par suite le caractère anisotrope de la relaxation, peuvent jouer un rôle essentiel; il en résulte dans certains cas des modifications profondes de la forme de la courbe fine. Nous étudierons également un cas où raisonner simplement dans le référentiel tournant n'est pas suffisant, celui où $\omega_{0}$ n'est pas proche de $\omega$, mais de $2 \omega$, et calculerons la forme de courbe de résonance obtenue.

C.1 INFLUENCE DES TERMeS DIAGONAUX DE LA RELAXATION RÉSONNANTE. - C.1.a Courbe large. Commençons par étudier le cas le plus simple, celui

$\left({ }^{13}\right)$ Il n'y a bien sûr aucune difficulté de principe à raisonner dans le référentiel du laboratoire comme nous l'avons fait plus haut lorsque le champ statique était supposé parfaitement homogène. Les expressions obtenues sont cependant peu maniables et nous ne les donnerons donc pas ici. Signalons toutefois un résultat de ce calcul qui permet de bien comprendre pourquoi le champ magnétique fictif ne joue dans le cas envisagé aucun rôle à résonance $\left(\omega_{0} \simeq \omega\right)$, ce qui pourrait a priori sembler étonnant puisque, dans le référentiel du laboratoire, les termes croisés entre inhomogénéités statique et oscillante donnent des composantes transversales de $\mathbf{V}$ oscillant à la pulsation $\omega$. En fait, si l'on effectue un calcul précis dans le référentiel du laboratoire de ces termes croisés, on trouve $K_{y z} \propto \cos \omega t, C_{z y} \propto \cos \omega t, S_{z y} \propto \sin \omega t$ (et des formules analogues où $y$ est remplacé par $x$ ). On voit alors sur (B.43b) que le champ transverse modulé est en fait un champ tournant à la vitesse angulaire - $\omega$, c'est-à-dire un champ antirésonnant; aucune des composantes modulées de $\mathbf{V}$ ne pouvant donc être séculaire, seule la partie tensorielle de la relaxation est effectivement importante. où le champ de radiofréquence est purement inhomogène et où les inhomogénéités du champ magnétique statique sont nulles. Comme nous l'avons déjà noté au $\S$ B.3.b. $\beta$, si l'on néglige les termes non résonnants de la matrice de relaxation $(R)$ [c'est-àdire les coefficients $K$ ] on trouve que la composante $M_{z}$ n'est pas couplée par $(R)$ à $M_{x}$ et $M_{y}$; l'effet de la radiofréquence inhomogène sur $M_{z}$ peut donc être caractérisé par la seule donnée du temps $T_{1}^{*}$ écrit en (B.27). Le raisonnement du § A.1 reste donc entièrement valable, la seule différence étant qu'il faut prendre la valeur exacte de $T_{1}^{*}$ au lieu de la valeur approchée (A.6). Au voisinage de $\omega_{0}=\omega$, les variations de $1 / T_{1}^{*}$ sont maintenant représentées, non par une seule courbe d'absorption de Lorentz, mais par la superposition d'une infinité de courbes d'absorption de largeurs différentes et dont les poids dépendent de la carte du champ de radiofréquence inhomogène ; en pratique, pourvu que cette carte de champ ne soit pas trop compliquée, la superposition des 3 ou 4 courbes de Lorentz correspondant aux temps $\tau_{n, l}$ les plus longs (c'est-à-dire aux largeurs les plus faibles) suffira largement pour rendre compte des résultats expérimentaux (on trouvera à la fin de l'appendice un tableau des expressions des $\tau_{n, l}$ pour les valeurs les plus basses de $n$ et $l$ ).

Calculons la valeur stationnaire $M_{z}^{\text {s }}$ de l'aimantation longitudinale. Avec les notations du $\S \mathrm{A}$, elle vaut :

$$
M_{z}^{\mathrm{s}}=M_{0} \frac{T_{\mathrm{r}}}{T_{\mathrm{r}}+T_{\mathrm{p}}}\left\{1-\frac{T_{1}^{0} / T_{1}^{*}}{1+T_{1}^{0} / T_{1}^{*}}\right\}
$$

La forme de courbe de résonance est donc donnée par la fonction :

$$
y\left(\Delta_{0}\right)=\left[1+\frac{T_{1}^{*}\left(\Delta_{0}\right)}{T_{1}^{0}}\right]^{-1}
$$

où $T_{1}^{*}\left(\Delta_{0}\right)$ est donné par (B.27). $\mathrm{Si}$, dans la sommation sur $n, l, m$ qui apparaît dans cette égalité, on ne retient qu'une valeur pour $n$ et une valeur pour $l$ (seul l'indice $m$ peut varier), le temps $\tau_{n, l}$ est fixé, et un calcul analogue à celui du $\S \mathrm{A}$ montre que $y\left(\Delta_{0}\right)$ est une fonction de Lorentz, de largeur :

$$
L=\frac{1}{\tau_{n, l}} \sqrt{1+T_{1}^{0} \tau_{n, l} \sum_{m}\left|\left\langle\varphi_{n, l, m} \mid \Omega_{1+}\right\rangle\right|^{2} / 4 V} .
$$

Par contre, si plusieurs modes de temps de diffusion $\tau_{n, l}$ différents interviennent effectivement, la fonction $y\left(\Delta_{0}\right)$ dépend de façon plus complexe de $\Delta_{0}$. Supposons pour fixer les idées que deux temps $\tau_{n, l}$, que nous noterons $\tau_{\mathrm{d}}$ et $\tau_{\mathrm{d}}^{\prime}$, interviennent dans $T_{1}^{*}$ :

$$
\frac{1}{T_{1}^{*}} \simeq \frac{k \tau_{\mathrm{d}}}{1+\left(\Delta_{0} \tau_{\mathrm{d}}\right)^{2}}+\frac{k^{\prime} \tau_{\mathrm{d}}^{\prime}}{1+\left(\Delta_{0} \tau_{\mathrm{d}}^{\prime}\right)^{2}}
$$


$k$ et $k^{\prime}$ étant des constantes proportionnelles au carré de l'intensité du champ de radiofréquence inhomogène. Alors :

$$
y\left(\Delta_{0}\right)=\frac{n\left(\Delta_{0}\right)}{\left[1+\left(\Delta_{0} \tau_{\mathrm{d}}\right)^{2}\right]\left[1+\left(\Delta_{0} \tau_{\mathrm{d}}^{\prime}\right)^{2}\right]+n\left(\Delta_{0}\right)}
$$

avec :

$n\left(\Delta_{0}\right)=k \tau_{\mathrm{d}} T_{1}^{0}\left[1+\left(\Delta_{0} \tau_{\mathrm{d}}^{\prime}\right)^{2}\right]+k^{\prime} \tau_{\mathrm{d}}^{\prime} T_{1}^{0}\left[1+\left(\begin{array}{ll}\Delta_{0} & \tau_{\mathrm{d}}\end{array}\right)^{2}\right]$.

Une décomposition en éléments simples de la fraction rationnelle (C.5) montre que $y\left(\Delta_{0}\right)$ est la superposition de deux fonctions de Lorentz, centrées en $\Delta_{0}=0 \quad\left(\omega_{0}=\omega\right) ;$ leurs largeurs, extrapolées à intensité de radiofréquence nulle $\left(k=k^{\prime}=0\right)$, sont simplement $1 / \tau_{d}$ et $1 / \tau_{d}^{\prime}$, mais, lorsque la radiofréquence n'est pas nulle, varient de façon plus complexe que (C.3). Donc, la présence de plusieurs modes de diffusion se traduit par le fait que la courbe de résonance (courbe large) est la superposition de plusieurs courbes de Lorentz au lieu d'une seulement.

C.1.b Courbe fine. - Que se passe-t-il maintenant si le champ de radiofréquence, au lieu d'être complètement inhomogène, possède également une partie homogène? Nous examinerons ici le cas plus général où la partie homogène a une fréquence quelconque $\omega^{\prime} / 2 \pi$ :

$$
\mathbf{B}_{1}^{\prime}(t)=-\frac{\omega_{1}^{\prime}}{\gamma} \cos \omega^{\prime} t
$$

(où $\omega_{1}^{\prime}$ est perpendiculaire à $O z$ ) en supposant, pour le moment, que $\omega^{\prime}$ et $\omega$ sont différents et que les termes non diagonaux de la relaxation sont non séculaires; le rôle de ces termes non diagonaux lorsque $\omega^{\prime} \simeq \omega$ sera examiné ensuite. Comme plus haut, nous supposerons que $\omega_{1}^{\prime} \tau_{1,1} \ll 1$, condition qui assure que la partie homogène du champ de radiofréquence ne perturbe pas la relaxation due à la partie inhomogène [cf. note $\left({ }^{4}\right)$ ]. Nous pouvons alors raisonner exactement comme dans le $\S \mathrm{A} .2$, et dire que l'effet du champ $\mathbf{B}_{1}^{\prime}(t)$ est d'effectuer la résonance magnétique habituelle sur un ensemble de spins dont les temps de relaxation $T_{1}$ et $T_{2}$, ainsi que la fréquence propre de précession, sont modifiés par les termes diagonaux de la relaxation produite par le champ inhomogène. A la courbe large calculée plus haut vient donc s'ajouter une courbe fine, les variations correspondantes de $M_{z}^{\text {s }}$ s'écrivant, avec les notations du $\S \mathrm{A}$ :

$$
\begin{aligned}
& M_{z}^{\mathrm{s}}=M_{0} \frac{T_{1}}{T_{\mathrm{p}}} \times \\
& \times\left\{1-\frac{\omega_{1}^{\prime 2} T_{1} / 4 T_{2}}{\left[\omega_{0}+\delta \omega_{0}^{*}-\omega^{\prime}\right]^{2}+\left(1 / T_{2}\right)^{2}+\omega_{1}^{\prime} T_{1} / 4 T_{2}}\right\} .
\end{aligned}
$$

Comme dans la formule (A.20), il faut noter que $T_{1}$, $T_{2}$ et $\delta \omega_{0}^{*}$ sont des fonctions de $\omega_{0}$, c'est-à-dire en fait de la valeur de $\omega^{\prime}$ choisie, puisque la courbe fine est située en $\omega_{0} \simeq \omega^{\prime}$ (les variations de $T_{1}^{*}, T_{2}^{*}$ et $\delta \omega_{0}^{*}$ en fonction de $\omega_{0}$ sont négligeables sur la largeur $l$ de la courbe fine et on peut remplacer $\omega_{0}$ par $\omega^{\prime}$ dans leurs expressions). Donc, plus $\omega^{\prime}$ est choisi proche de $\omega$, plus l'effet de la relaxation résonnante sur la courbe fine (élargissement, diminution de la hauteur) est marqué. On note que, parmi ces effets, figure un déplacement $\delta \omega_{0}^{*}$ de la position de la résonance fine ; $\delta \omega_{0}^{*}$ varie comme une superposition de courbes de dispersion en fonction de $\omega^{\prime}-\omega$ [cf. (B.39) où $\omega_{0}$ a été remplacé par $\left.\omega^{\prime}\right]$ : le déplacement est maximal lorsque $\omega^{\prime}-\omega= \pm 1 / \tau_{\mathrm{d}}\left(\tau_{\mathrm{d}}\right.$ étant le temps $\tau_{n, l}$ dont le poids dans $\delta \omega_{0}^{*}$ est le plus grand), nul si $\omega^{\prime}=\omega$, et décroît en $1 /\left(\omega^{\prime}-\omega\right)$ lorsque $\left|\omega^{\prime}-\omega\right| \gg 1 / \tau_{\mathrm{d}}$ (dans ce cas, $\delta \omega_{0}^{*}$ peut devenir grand devant l'élargissement de la courbe fine dû au champ de radiofréquence inhomogène; cf. $\S$ B.3.a. $\beta$ ).

Nous avons déjà noté que le déplacement $\delta \omega_{0}^{*}$ est lié aux transitions virtuelles induites par le champ magnétique inhomogène agissant sur les spins et au couplage qu'elles induisent entre leurs deux niveaux d'énergie. Dans le référentiel tournant, le champ statique a une intensité $\left(\omega-\omega_{0}\right) / \gamma$, et les énergies des niveaux non perturbés sont $\pm \hbar\left(\omega_{0}-\omega\right) / 2$. Le couplage dû aux transitions virtuelles fait que ces deux niveaux se repoussent, leurs énergies devenant $\pm \hbar\left(\omega_{0}-\omega+\delta \omega_{0}^{*}\right)$ [d'après (B.39), $\delta \omega_{0}^{*}$ a le même signe que $\left.\omega_{0}-\omega\right]$. En d'autres termes, l'effet du déplacement $\delta \omega_{0}^{*}$ est celui d'un champ magnétique fictif venant s'ajouter, avec le même signe, au champ efficace dans le référentiel tournant. Pour la courbe étroite, ceci se traduit par le fait que son centre est situé à une valeur du champ magnétique statique différente de $-\omega^{\prime} / \gamma$, le centre de la courbe étroite étant repoussé par celui de la courbe large.

En toute rigueur, il faut noter que la formule (C.8) n'est pas valable lorsque $\omega^{\prime}=\omega$, c'est-à-dire lorsque la courbe fine est centrée sur le sommet de la courbe large. En effet, nous n'avons retenu que les termes diagonaux de la relaxation et, si l'on choisit $\omega^{\prime}=\omega$ (c'est-à-dire si l'on fait varier $\omega_{0}$ au voisinage de $\omega$ ), la matrice $(R)$ contient deux éléments $T_{x y}$ et $T_{y x}$ non diagonaux, qui sont à la fois séculaires et résonnants. Aussi faudrait-il, pour obtenir la forme exacte de la courbe fine, reprendre le calcul des équations de Bloch dans le cas où il existe un terme de couplage entre $M_{x}$ et $M_{y}$ dû à la relaxation. Comme c'est précisément ce que nous allons faire dans le paragraphe suivant, nous ne donnons pas ici ce calcul; signalons toutefois dès maintenant que les termes $T_{x y}$ et $T_{y x}$ peuvent changer la largeur et la hauteur de la courbe fine, mais ni sa position, ni sa forme d'absorption lorentzienne.

C. 2 INFLUENCE DES TERMES NON DIAGONAUX ; EFFETS DE LA RELAXATION ANISOTROPE. - C.2.a Equation d'évolution de l'aimantation. - Lorsque $\omega_{0} \simeq \omega$, 
nous avons vu plus haut que certains termes non diagonaux de la matrice de relaxation $(R)$ peuvent devenir séculaires; nous allons envisager ici le cas où le champ statique est inhomogène, ce qui entraîne (cf. $\S$ B.3.c) que $M_{z}$ peut être couplé par $(R)$ à $M_{x}$ et $M_{y}$. Seules les parties de cette matrice de relaxation qui sont à la fois résonnantes et séculaires seront prises en compte dans les calculs qui vont suivre ; dans ce but, nous raisonnerons directement dans le référentiel tournant $O X Y Z$ ( $O X Y Z$ tourne autour de $O Z=O z$ à la vitesse angulaire $\omega$ ), en négligeant les composantes de l'inhomogénéité qui ne sont pas statiques dans ce référentiel. De plus, nous supposerons que $\left|\Delta_{0}\right|=\left|\omega_{0}-\omega\right| \ll 1 / \tau_{1,1}$ (on se place au centre de la courbe large). En passant dans le référentiel tournant, nous voyons que les coefficients $S$ sont nuls et que les coefficients $K$ et $C$ prennent la même valeur $A$ donnée par :

$$
\begin{aligned}
A_{X_{i} X_{j}} & =\int_{0}^{\infty} \mathrm{d} \tau \overline{\Omega_{1 X_{i}}[\mathrm{r}(t)] \Omega_{1 X_{j}}[\mathbf{r}(t-\tau)]} \\
& =\sum_{n, l, m}\left\langle\Omega_{1_{X_{i}}} \mid \varphi_{n, l, m}\right\rangle\left\langle\varphi_{n, l, m} \mid \Omega_{1 X_{j}}\right\rangle \frac{\tau_{n, l}}{V}
\end{aligned}
$$

avec $X_{i}, X_{j}=X, Y$ ou $Z$. Rappelons [cf. (B. $49 a$ et $c$ ] que, dans cette égalité, ce sont les composantes $\Omega_{1 X_{i}}$ et $\Omega_{1 X_{j}}$ de l'inhomogénéité statique dans le référentiel tournant qui interviennent; si $X_{i}=Z$, cette composante est égale, en chaque point $\mathbf{r}$, à la valeur de la composante sur $O z$ de l'inhomogénéité statique dans le référentiel du laboratoire ; si $X_{i}=X$ ou $Y$, elle est égale en chaque point $\mathbf{r}$ à la composante sur $O x$ ou $O y$ de l'inhomogénéité modulée, divisée par deux. On obtient alors :

$(R)=\left[\begin{array}{ccc}-A_{Y Y}-A_{Z Z} & A_{X Y} & A_{X Z} \\ A_{X Y} & -A_{X X}-A_{Z Z} & A_{Y Z} \\ A_{X Z} & A_{Y Z} & -A_{X X}-A_{Y Y}\end{array}\right]$.

Cette matrice est symétrique, ce qui était prévisible a priori (nous avons vu plus haut que le champ magnétique fictif est nul à résonance).

D'autres processus d'évolution peuvent influencer l'aimantation globale $\mathbf{M}$ des atomes. A la relaxation due au champ de radiofréquence inhomogène peut s'ajouter une relaxation due à d'autres processus, que nous caractériserons par un temps longitudinal $T_{1}^{0}$ et un temps transversal $T_{2}^{0}$. Ces temps de relaxation incorporent l'effet du pompage optique sur les atomes qui, de plus, ajoute un terme source $M_{0} / T_{\mathrm{p}}$ dans l'équation d'évolution de $M_{z}$. Enfin, nous tenons compte de l'effet d'un champ de radiofréquence homogène parallèle à $O x\left({ }^{14}\right)$ d'intensité

$\left({ }^{14}\right)$ Nous définissons les axes transversaux $O X$ et $O Y$ du référentiel tournant en chaque point $\mathbf{r}$ par le fait que $O X$ coïncide à l'instant $t=0$ (instant où le champ linéaire est maximal) avec l'axe $O x$ du laboratoire, parallèle à la partie homogène du champ de radiofréquence. $-\left(\omega_{1} / \gamma\right) \cos \omega t$ [partie homogène du champ de radiofréquence] qui vient s'ajouter au champ statique $\mathbf{B}_{0}$. Dans ces conditions, l'équation d'évolution de la matrice $(M)$ de composantes $M_{X}, M_{Y}$ et $M_{Z}$ s'écrit :

$$
\begin{aligned}
& \frac{\mathrm{d}}{\mathrm{d} t}(M)= \\
& =\left\{-\left[\begin{array}{c}
1 / T_{2}^{0} \\
1 / T_{2}^{0} \\
1 / T_{1}^{0}
\end{array}\right]+(R)+\left[\begin{array}{ccc}
0 & -\Delta_{0} & 0 \\
\Delta_{0} & 0 & -\omega_{1} / 2 \\
0 & \omega_{1} / 2 & 0
\end{array}\right]\right\}(M)+ \\
& +\frac{1}{T_{\mathrm{p}}}\left[\begin{array}{c}
0 \\
0 \\
M_{0}
\end{array}\right]
\end{aligned}
$$

[nous négligeons l'effet de la partie antirésonnante du champ de radiofréquence].

Remarque. - Les coefficients $A_{X_{i} X_{i}}$ ne sont rien d'autre que la densité spectrale, prise à fréquence nulle, de la fonction aléatoire $\Omega_{1 X_{i}}[\mathrm{r}(t)]$; ils sont donc positifs ou éventuellement nuls [le processus aléatoire qui régit le mouvement des atomes est supposé ici stationnaire] ; ceci se vérifie d'ailleurs sur la deuxième égalité (C.9). Par contre, les coefficients $A_{X_{i} X_{j}}$ n'ont pas, a priori, de signe bien déterminé : ils peuvent être nuls si les variations aléatoires $\Omega_{1 X_{i}}$ et $\Omega_{1 X_{j}}$ sont incorrélées, positifs ou négatifs si elles sont corrélées. On démontre cependant aisément (inégalité de Schwarz) que :

$$
\left[A_{X_{i} X_{j}}\right]^{2} \leqslant A_{X_{i} X_{i}} \cdot A_{X_{j} X_{j}}
$$

l'égalité n'étant réalisée que si $\Omega_{1 X_{i}}(\mathbf{r})$ est proportionnelle en tout point $\mathbf{r}$ à $\Omega_{1 X_{j}}[\mathbf{r}]$.

C.2.b Valeur stationnaire de l'aimantation. - Introduisons les notations :

$$
\left\{\begin{array}{l}
\Gamma_{X}=\frac{1}{T_{2}^{0}}+A_{Y Y}+A_{Z Z} \\
\Gamma_{Y}=\frac{1}{T_{2}^{0}}+A_{Z Z}+A_{X X} \\
\Gamma_{Z}=\frac{1}{T_{1}^{0}}+A_{X X}+A_{Y Y}
\end{array}\right.
$$

Les valeurs stationnaires des trois composantes de $(M)$ sont alors données par :

$$
\begin{aligned}
& \left(\begin{array}{ccc}
\Gamma_{X} & -A_{X Y}+\Delta_{0} & -A_{X Z} \\
-A_{X Y}-\Delta_{0} & \Gamma_{Y} & -A_{Y Z}+\omega_{1} / 2 \\
-A_{X Z} & -A_{Y Z}-\omega_{1} / 2 & \Gamma_{Z}
\end{array}\right) \times \\
& \times\left(\begin{array}{c}
M_{X} \\
M_{Y} \\
M_{Z}
\end{array}\right)=\left(\begin{array}{c}
0 \\
0 \\
M_{0} / T_{\mathrm{p}}
\end{array}\right) .
\end{aligned}
$$


Le déterminant du système linéaire ainsi obtenu vaut :

$$
\begin{aligned}
D\left(\Delta_{0}\right)=\Gamma_{Z}\left[\Delta_{0}\right. & \left.-\omega_{1} A_{X Z} / 2 \Gamma_{Z}\right]^{2}+\left(\frac{\omega_{1}}{2}\right)^{2}\left[\Gamma_{X}-A_{X Z}^{2} / \Gamma_{Z}\right] \\
& +\Gamma_{X} \Gamma_{Y} \Gamma_{Z}-2 A_{X Y} A_{Y Z} A_{X Z} \\
& \left.-\Gamma_{X} A_{Y Z}^{2}-\Gamma_{Y} A_{X Z}^{2}-\Gamma_{Z} A_{X Y}^{2} \cdot \quad \text { (C. } 15\right)
\end{aligned}
$$

La valeur stationnaire $M_{Z}^{\mathrm{s}}$ de $M_{Z}$ est :

$$
\begin{aligned}
M_{Z}^{\text {s }} & =\frac{M_{0}}{T_{\mathrm{p}} \Gamma_{Z}} \frac{N\left(\Delta_{0}\right)}{D\left(\Delta_{0}\right)} \\
& =\frac{M_{0}}{T_{\mathrm{p}} \Gamma_{Z}}\left[1-Q\left(\Delta_{0}\right) / D\left(\Delta_{0}\right)\right]
\end{aligned}
$$

avec :

$$
N\left(\Delta_{0}\right)=\Gamma_{Z}\left[\Delta_{0}^{2}+\Gamma_{X} \Gamma_{Y}-A_{X Y}^{2}\right]
$$

et :

$$
\begin{aligned}
& Q\left(\Delta_{0}\right)=\left(\frac{\omega_{1}}{2}\right)^{2}\left[\Gamma_{X}-2 A_{X Z}^{2} / \Gamma_{Z}\right]- \\
& \quad-\omega_{1} A_{X Z}\left[\Delta_{0}-\omega_{1} A_{X Z} / 2 \Gamma_{Z}\right] \\
& \quad-\Gamma_{X} A_{Y Z}^{2}-\Gamma_{Y} A_{X Z}^{2}-2 A_{X Y} A_{Y Z} A_{X Z} .
\end{aligned}
$$

Les variations de $M_{Z}^{s}$ en fonction du champ magnétique, c'est-à-dire de $\Delta_{0}$, donnent donc une courbe obtenue par superposition d'une courbe d'absorption et d'une courbe de dispersion; toutes deux sont centrées, non en $\Delta_{0}=0$, mais en $\Delta_{0}=\delta \tilde{\omega}$, avec :

$$
\begin{aligned}
\delta \tilde{\omega} & =\omega_{1} A_{X Z} / 2 \Gamma_{Z} \\
& =\frac{\omega_{1}}{2} \frac{A_{X Z}}{A_{X X}+A_{Y Y}+1 / T_{1}^{0}} .
\end{aligned}
$$

Leurs largeurs $\mathfrak{L}$ sont données par :

$$
\begin{aligned}
& \mathfrak{L}^{2}=\frac{1}{\Gamma_{Z}}\left\{\left(\frac{\omega_{1}}{2}\right)^{2}\left[\Gamma_{X}-A_{X Z}^{2} / \Gamma_{Z}\right]+\Gamma_{X} \Gamma_{Y} \Gamma_{Z}-\right. \\
& \left.-2 A_{X Y} A_{Y Z} A_{X Z}-\Gamma_{X} A_{Y Z}^{2}-\Gamma_{Y} A_{X Z}^{2}-\Gamma_{Z} A_{X Y}^{2}\right\} .
\end{aligned}
$$

La hauteur de la courbe d'absorption vaut :

$$
\begin{aligned}
h_{\mathrm{a}}=\frac{\ddot{M}_{0}}{\Gamma_{Z} T_{\mathrm{p}}} & \times \frac{1}{\Gamma_{Z} \mathfrak{L}^{2}} \times \\
& \times\left\{\left(\omega_{1} / 2\right)^{2}\left[\Gamma_{X}-2 A_{X Z}^{2} / \Gamma_{Z}\right]-\right. \\
& \left.-\Gamma_{X} A_{Y Z}^{2}-\Gamma_{Y} A_{Z X}^{2}-2 A_{X Y} A_{Y Z} A_{Z X}\right\}
\end{aligned}
$$

et celle de la courbe de dispersion :

$$
h_{\mathrm{d}}=\frac{M_{0}}{T_{\mathrm{p}} \Gamma_{Z}} \frac{\omega_{1} A_{X Z}}{2 \Gamma_{Z} \mathcal{L}} .
$$

Les résultats que nous obtenons sont donc très différents de ceux que donnent les classiques équations de Bloch qui, lorsqu'on s'intéresse à la valeur stationnaire de $M_{z}$, prévoient une courbe de résonance lorentzienne, centrée en $\Delta_{0}=0$, en forme d'absorption pure.

Le même calcul donnerait aussi bien les valeurs stationnaires $M_{X}^{\mathbf{s}}$ et $\boldsymbol{M}_{Y}^{\mathbf{s}}$ des composantes transversales de l'orientation. On obtiendrait alors des courbes d'absorption et de dispersion, centrées cette fois encore en $\Delta_{0}=\delta \tilde{\omega}_{0}$, mais avec des hauteurs différentes.

C.2.c Discussion. - C.2.c. $\alpha$ Cas où le champ de radiofréquence est purement inhomogène; renversement de la courbe fine. - Dans les formules obtenues plus haut, faisons $\omega_{1}=0$; il vient :

$h_{\mathrm{a}}\left(\omega_{1}=0\right)=-\frac{M_{0}}{\Gamma_{Z} T_{\mathrm{p}}} \frac{\Gamma_{X} A_{Y Z}^{2}+\Gamma_{Y} A_{X Z}^{2}+2 A_{X Y} A_{Y Z} A_{Z X}}{\Gamma_{Z} \mathfrak{L}^{2}\left(\omega_{1}=0\right)}$

$$
\begin{aligned}
\mathfrak{L}^{2}\left(\omega_{1}=0\right)= & \frac{1}{\Gamma_{Z}}\left\{\Gamma_{X} \Gamma_{Y} \Gamma_{Z}-2 A_{X Y} A_{Y Z} A_{X Z}-\right. \\
& \left.-\Gamma_{X} A_{Y Z}^{2}-\Gamma_{Y} A_{X Z}^{2}-\Gamma_{Z} A_{X Y}^{2}\right\} .
\end{aligned}
$$

Quant à $h_{\mathrm{d}}$ et $\delta \tilde{\omega}$, ils sont nuls. Cependant, à l'opposé des résultats obtenus au $\S$ C.1 (cas d'un champ statique homogène), nous trouvons ici qu'une courbe fine est présente même lorsque $\omega_{1}$ est nul. En fait, la formule (C.22a) indique que la courbe fine n'existe que si $A_{Y Z}, A_{X Z} \neq 0$, c'est-à-dire si $O Z$ n'est pas un axe principal de l'ellipsoïde de relaxation $\left({ }^{15}\right)$; cette courbe fine est donc étroitement liée à l'anisotropie de la relaxation provenant des corrélations entre composantes des inhomogénéités. On vérifie de plus que son existence tient à la présence simultanée d'inhomogénéités du champ statique et de radiofréquence (les coefficients $A_{X Z}$ et $A_{Y Z}$ sont des termes croisés, linéaires séparément par rapport à ces deux inhomogénéités). Le fait le plus remarquable est cependant que la hauteur $h_{\mathrm{a}}\left(\omega_{1}=0\right)$ est négative $\left({ }^{16}\right)$ (nous supposons que $M_{0}$ est positif): l'orientation $M_{z}^{\mathbf{s}}$, au lieu d'être minimale lorsque $\omega_{0}=\omega$, comme habituellement en résonance magnétique, est maximale $\left(\left|M_{z}^{\text {s }}\right|\right.$ est supérieure à la valeur $\left|M_{0}\right| T_{\mathrm{p}} / \Gamma_{Z}$ qui serait obtenue en l'absence de courbe fine). La courbe de résonance a donc l'allure

( $\left.{ }^{15}\right)$ En pratique, pour que $A_{X Z}$ par exemple ne soit pas nul, il faut choisir certaines configurations des inhomogénéités magnétiques sur la cellule. En effet, la formule (C.9) montre que $A_{X Z}$ est nul si les perturbations aléatoires $\Omega_{1 X}[\mathbf{r}(t)]$ et $\Omega_{12}[\mathbf{r}(t)]$ vues par un atome donné sont non corrélées, et la seconde des égalités (C.9) permet de calculer leurs corrélations en fonction des variations spatiales des inhomogénéités. Les dispositifs expérimentaux qui permettent d'obtenir une forte corrélation entre les diverses composantes de l'inhomogénéité, et par suite une grande hauteur $h_{\mathrm{a}}$, seront discutés dans la deuxième partie de cet article.

${ }^{16}$ ) On peut voir immédiatement sur (C.22a) que $h_{\mathrm{a}}<0$ en choisissant les axes $O X$ et $O Y$ de façon que $A_{X Z}=0$; ce choix des axes est ici possible puisque la partie homogène du champ de radiofréquence est supposée nulle (cf. note $\left({ }^{14}\right)$ ). 


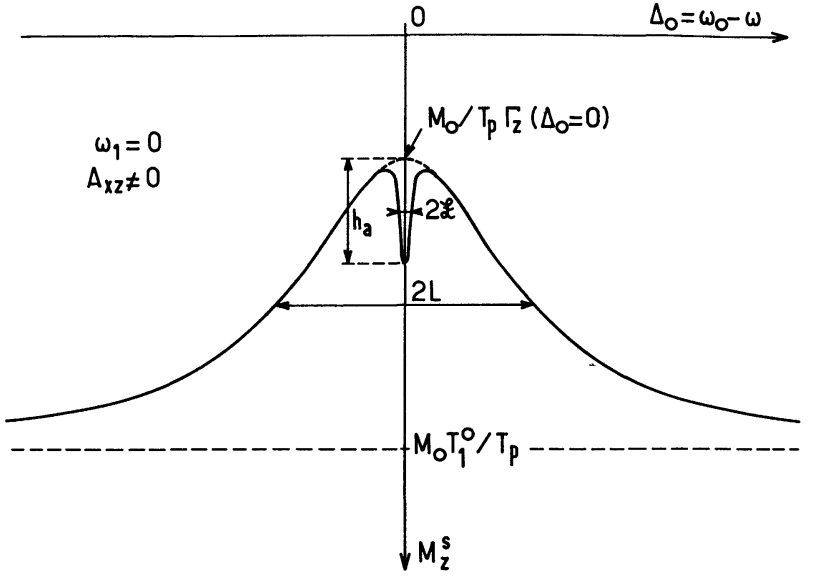

FIG. 2. - Courbe de résonance obtenue avec un champ de radiofréquence parfaitement inhomogène $\left(\omega_{1}=0\right)$, en présence d'inhomogénéités du champ statique. Lorsque les variations des composantes transversales du champ de radiofréquence et longitudinąle de l'inhomogénéité statique sont corrélées $\left(A_{X Z} \neq 0\right)$, il apparaît au sommet de la courbe large une courbe fine renversée, de hauteur $h_{\mathrm{a}}: M_{z}^{\mathrm{s}}$ est maximal à résonance (si $A_{X Z}$ était nul, la courbe large apparaîtrait seule, sans courbe fine).

de la figure 2 : pour les valeurs de $\Delta_{0}$ comparables à $1 / \tau_{n, l}$, on obtient la même courbe large que plus haut, l'anisotropie de la relaxation donnant des termes non séculaires dans l'évolution de $\mathbf{M}$ qui n'ont aucun effet notable; par contre, pour les petites valeurs de $\Delta_{0}$, on voit apparaître la courbe fine renversée et, à résonance, on assiste à une reconstruction d'une partie de l'orientation détruite par la courbe large [cet effet se produit lorsque $\left|\Delta_{0}\right| \lesssim \mathcal{L}\left(\omega_{1}=0\right)$, la largeur $\mathcal{L}\left(\omega_{1}=0\right)$ étant donnée par (C.22.b)].

Remarque. - En utilisant (C.12) on montre par un calcul sans difficultés que le second membre de (C.22.b) est strictement positif, quelles que soient , les variations spatiales et l'intensité des inhomogénéités magnétiques. Lorsque le champ de radiofréquence inhomogène est nul, la largeur $\mathfrak{L}\left(\omega_{1}=0\right)$ vaut $1 / T_{2}^{0}$, comme en résonance magnétique ordinaire : la largeur $\mathfrak{L}\left(\omega_{1}=0\right)$ varie de façon notable, et la hauteur de la courbe fine renversée est à misaturation lorsque $A_{X Z}$ ou $A_{Y Z}$ est de l'ordre de $1 / T_{2}^{0}$.

C. 2.c. $\beta$ Courbe de dispersion et déplacement de la résonance dus à la partie homogène du champ de radiofréquence. - Lorsque $\omega_{1}$ n'est pas nul, il faut prendre les formules complètes (C.18), (C.19), (C.20) et (C.21). Tout d'abord, on remarque alors la présence du déplacement de fréquence $\delta \widetilde{\omega}\left({ }^{17}\right)$, proportionnel à l'intensité $\omega_{1}$ du champ de radiofréquence homogène. Il s'agit là d'un fait assez remarquable : habituellement, les déplacements de fréquences en résonance magnétique sont des fonctions paires de $\omega_{1}$

$\left({ }^{17}\right) \delta \tilde{\omega}$ est bien sûr complètement distinct du déplacement $\delta \omega_{0}^{*}$ étudié plus haut. qui restent inchangées si $\omega_{1}$ change de signe, c'est-àdire si on change de $\pi$ la phase du champ de radiofréquence; physiquement, ceci tient au fait que, si le seul phénomène périodique est l'oscillation du champ de radiofréquence, sa phase ne peut intervenir dans l'absolu. Ici, c'est la phase relative des deux champs de radiofréquence (homogène et inhomogène) qui intervient : si $\omega_{1}$ change de signe, le champ de radiofréquence homogène change de position relative (dans le référentiel tournant) par rapport à l'ellipsoïde de relaxation et à l'aimantation initiale $\mathbf{M}_{0}$, et le problème physique est évidemment différent. D'ailleurs, le déplacement $\delta \tilde{\omega}$ est proportionnel à $A_{X Z} ; \delta \tilde{\omega}$ est donc nul s'il n'y a pas de termes en $X Z$ dans l'équation de l'ellipsoïde de relaxation, c'est-àdire si les axes $O X$ (qui porte le champ de radiofréquence homogène) et $O Z$ sont les axes de l'ellipse intersection de $X O Z$ par cet ellipsoïde ; ceci montre encore l'importance des dispositions relatives dans le référentiel tournant de cet ellipsoïde et du champ tournant homogène. Cette disposition relative dépend des cartes sur la cellule des champs inhomogènes statique et oscillant [voir note $\left({ }^{15}\right)$ ] ; évidemment, elle varie également si l'on change la phase relative des parties homogène et inhomogène du champ de radiofréquence.

L'autre phénomène nouveau qui apparaît est la présence d'une courbe de dispersion; la courbe de résonance est donc, dans ce cas, asymétrique $\left(M_{z}^{\text {s }}\right.$ ne reste pas invariant si l'on change $\Delta_{0}-\delta \tilde{\omega}$ en son opposé), ce qui est également inhabituel dans les variations de la composante longitudinale de l'aimantation. On peut faire les mêmes remarques sur cette courbe de dispersion que sur le déplacement $\delta \widetilde{\omega}$, tous deux étant proportionnels à $A_{X Z}$.

La largeur $\mathcal{L}$ est une fonction croissante de $\omega_{1}$ [cf. (C. 19)], car :

$$
\Gamma_{X} \Gamma_{Z} \geqslant A_{X Z}^{2}
$$

[cette inégalité découle immédiatement de (C.12)]; la formule (C.19) présente d'ailleurs une certaine analogie avec celle qui donne la largeur en résonance magnétique ordinaire. Quant à la hauteur $h_{\mathrm{a}}$, c'est une fonction de $\omega_{1}$, telle que, lorsque $\omega_{1}$ devient très grand :

$$
h_{\mathrm{a}} \underset{\omega_{1} \rightarrow \infty}{\longrightarrow} \frac{M_{0}}{\Gamma_{Z} T_{\mathrm{p}}} \frac{\Gamma_{X} \Gamma_{Z}-2 A_{X Z}^{2}}{\Gamma_{X} \Gamma_{Z}-A_{X Z}^{2}}
$$

valeur qui, suivant les cas, peut être positive ou négative. La figure 3 montre les variations de $h_{\mathrm{a}}$ en fonction de $\omega_{1}$ dans différentes situations possibles.

Les formes de courbe de résonance obtenues dans ce cas dépendent donc de façon critique de la valeur du coefficient $A_{X Z}$. Si les variations spatiales des inhomogénéités sont telles que $A_{X Z}=0\left[\Omega_{1 X}\right.$ et $\Omega_{1 Z}$ sont incorrélées], la situation n'est pas très différente de celles étudiées aux $\S \S$ C.1.b ou C.2.c. $\alpha$ précédents (courbe d'absorption, pas de déplacement); 


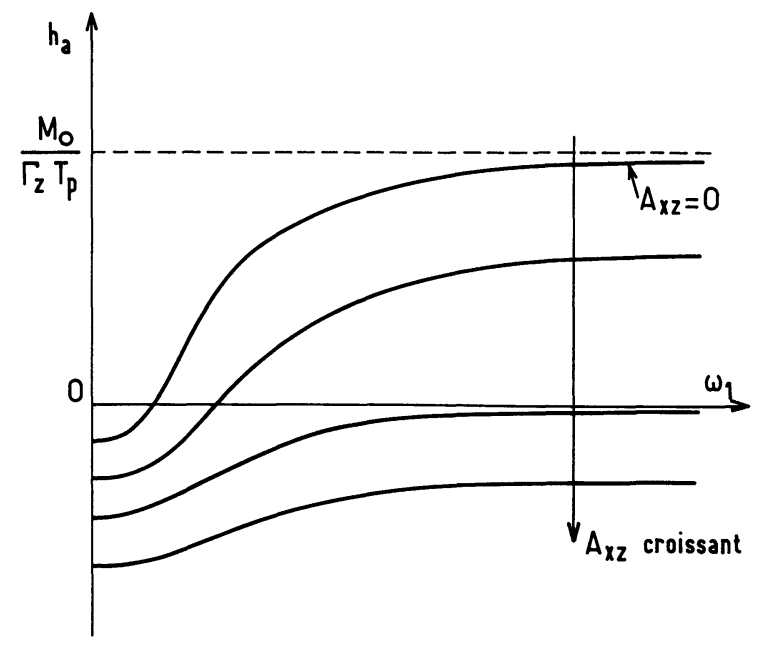

FIG. 3. - Variations de la hauteur $h_{\mathrm{a}}$ de la courbe d'absorption (courbe fine) en fonction de l'intensité $\omega_{1}$ du champ de radiofréquence homogène (celles de la radiofréquence inhomogène et du champ inhomogène statique étant supposées constantes). Si $A_{X Z}=0, h_{\text {a }}$ part d'une valeur négative (ou nulle si $A_{Y Z}$ est aussi nul) et croît ensuite en fonction de $\omega_{1}$, pour atteindre la valeur positive $M_{0} / \Gamma_{Z} T_{\mathrm{p}}$ (orientation totalement détruite à saturation). Si $A_{X Z}$ n'est pas nul, $h_{\text {a }}$ peut, lorsque $\omega_{1} \rightarrow \infty$ tendre vers zéro ou même une valeur négative [formule (C.24)].

en effet, lorsque $\omega_{1}$ prend des valeurs de plus en plus grandes, on passe progressivement d'une situation où la courbe fine d'absorption est renversée (à moins que $A_{Y Z}$ soit aussi nul) à celle où elle a le signe habituel (cf. Fig. 3); à la limite où $\omega_{1} \rightarrow \infty$, le fait que $h_{\mathrm{a}} \rightarrow M_{0} / \Gamma_{Z} T_{\mathrm{p}}$ indique que toute l'orientation qui avait subsisté au sommet de la courbe large est détruite. $\mathrm{Si}$, au contraire, $A_{X Z}$ prend des valeurs importantes [corrélation forte entre $\Omega_{1 X}$ et $\Omega_{1 z}$ ], la situation est nettement différente. Tout d'abord, pour certaines valeurs de $A_{X Z}, h_{\mathrm{a}}$ peut tendre vers zéro si $\omega_{1} \rightarrow \infty$, ou même vers une valeur négative; de plus, $h_{\mathrm{d}}$ et $\delta \widetilde{\omega}$ ne sont cette fois pas nuls : la résonance fine est déplacée et elle comprend une courbe de dispersion (les deux.composantes, absorption et dispersion, étant toutes deux centrées au même point). $\delta \widetilde{\omega}$ varie linéairement en fonction de $\omega_{1} ; h_{\mathrm{d}}$ commence par croître linéairement puis, lorsque $\omega_{1} \rightarrow \infty$, tend vers une valeur limite (saturation). Les variations de $h_{\mathrm{d}}$ en fonction de $\omega_{1}$ sont représentées sur la figure 4 . La figure 5 montre l'allure de la courbe de résonance obtenue dans un cas où $h_{\mathrm{a}}$ est négligeable devant $h_{\mathrm{d}}$.

Remarque. - En utilisant (C.12), on montre aisément que :

$$
\left\{\begin{array}{l}
D\left(\Delta_{0}\right) \geqslant 0 \\
D\left(\Delta_{0}\right)-Q\left(\Delta_{0}\right)=\Gamma_{Z}\left[\Delta_{0}^{2}+\Gamma_{X} \Gamma_{Y}-A_{X Y}^{2}\right] \geqslant 0
\end{array}\right.
$$

inégalités qui indiquent que $M_{Z}^{\text {s }}$ est toujours du même signe que $M_{0}$. De même, on montre par le calcul que :

$$
D\left(\Delta_{0}\right)-\frac{1}{T_{1}^{0} \Gamma_{Z}} N\left(\Delta_{0}\right) \geqslant 0 .
$$

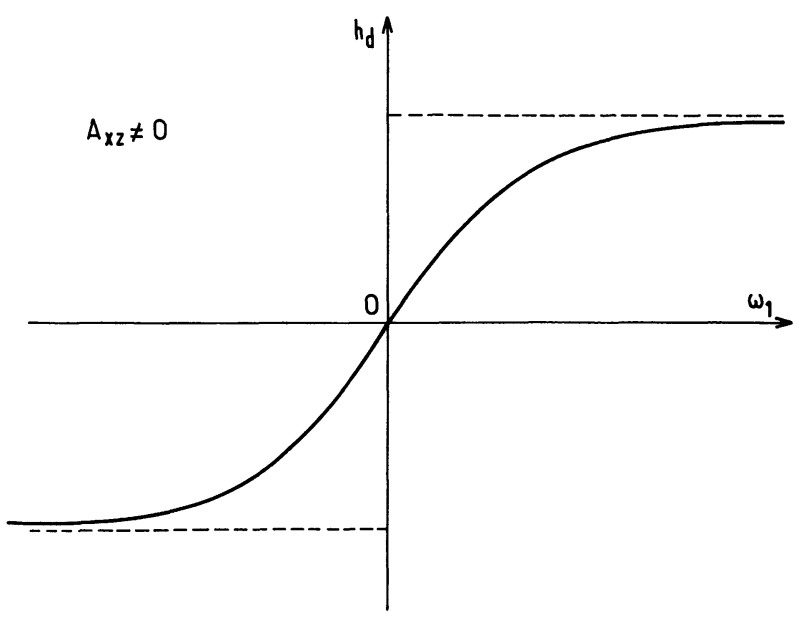

Fig. 4. - Variations de la hauteur $h_{\mathrm{d}}$ de la courbe de dispersion (courbe fine) en fonction de $\omega_{1}$ (on a supposé $A_{X Z} \neq 0$, pour que $h_{\mathrm{d}}$ ne soit pas nulle); $h_{\mathrm{d}}$ est une fonction impaire de $\omega_{1}$ : la dispersion change de signe si l'on renverse la phase de la partie homogène du champ de radiofréquence.

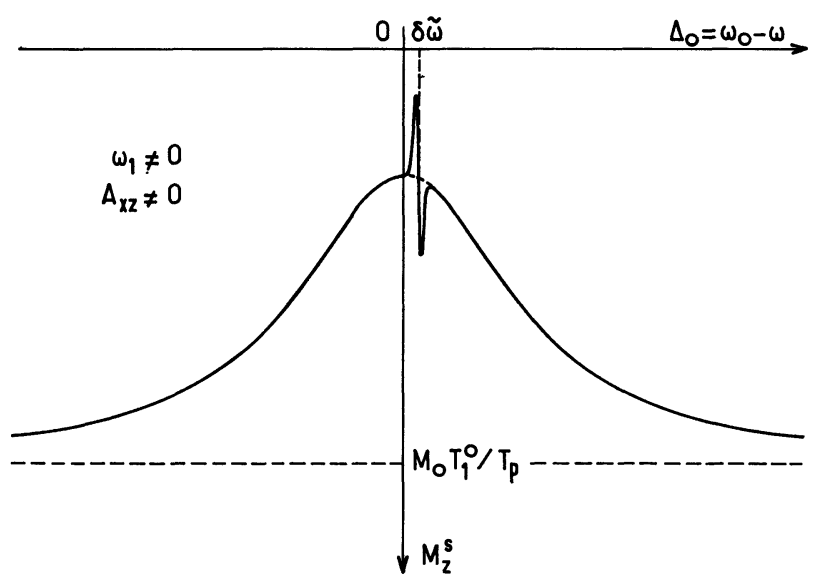

FIG. 5. - Modifications de la figure 2 dues à la présence d'une partie homogène du champ de radiofréquence. On a représenté le cas où la courbe fine est pratiquement une dispersion pure; cette courbe subit un déplacement $\delta \widetilde{\omega}$, proportionnel à l'intensité $\omega_{1}$ de la partie homogène du champ de radiofréquence.

Par suite :

$$
0 \leqslant \frac{M_{Z}^{\mathrm{s}}}{M_{0}} \leqslant \frac{T_{1}^{0}}{T_{\mathrm{p}}} .
$$

L'effet des deux champs de radiofréquence (homogène et inhomogène) se traduit donc toujours par une diminution de l'orientation stationnaire obtenue, mais jamais par un changement de signe ; par suite, les courbes de résonance des figures 2 et 5 ne peuvent traverser ni l'axe horizontal $M_{Z}=0$ ni l'asymptote $M_{Z}=M_{0} T_{1}^{0} / T_{\mathrm{p}}$ de la courbe large.

C.2.c. $\gamma$ Interprétation géométrique. - Comment comprendre physiquement les résultats précédents concernant les formes de courbes de résonance? Nous avons vu que les effets les plus nouveaux se produisent lorsque $A_{X Z} \neq 0$; ce sont donc $\Omega_{1 X}$ 
(composante de l'inhomogénéité oscillante sur l'axe $O X$ parallèle au champ homogène oscillant) et $\Omega_{1 Z}$ (composante de l'inhomogénéité statique sur $O Z$ ), qui jouent un rôle critique (ou, plus précisément, leurs corrélations). Pour raisonner dans un cas simple, nous allons supposer que $\Omega_{1 Y}$ est nul en tout point $r$, et que :

$$
\Omega_{1 Z}(\mathbf{r})=\lambda \Omega_{1 X}(\mathbf{r})
$$

(les variations de $\Omega_{1 X}$ et $\Omega_{1 Z}$ sont alors complètement corrélées) ; en d'autres termes, l'inhomogénéité dans le référentiel tournant se présente comme un vecteur de direction $O \mathbf{u}$ fixe mais de longueur variable $\left({ }^{\mathbf{1 8}}\right)$. $O \mathbf{u}$ appartient au plan $X O Z$ et fait avec $O X$ un angle $\theta$ tel que :

$$
\operatorname{tg} \theta=\lambda
$$

On a alors :

$$
\begin{aligned}
& A_{Z Z}=\lambda^{2} A_{X X} \\
& A_{X Z}=\lambda A_{X X} .
\end{aligned}
$$

On montre dans ce cas que l'ellipsoïde de relaxation est dégénéré en un cylindre de révolution d'axe $O \mathbf{O u}$. La direction $O \mathbf{u}$ correspond à une direction propre de la relaxation de valeur propre nulle, ce qui signifie que la composante $M_{u}$ de l'aimantation n'est pas affectée par la relaxation magnétique (physiquement, ceci se comprend bien puisque l'inhomogénéité dans le référentiel tournant reste parallèle à $O \mathbf{u}$ en tout point r). Quant aux directions du plan perpendiculaire à $O \mathbf{u}$, ce sont des directions de relaxation maximale.

Commençons par supposer que $\omega_{1}=0$; lorsque $\omega_{0}$ varie, le champ efficace $\mathbf{B}_{\text {eff }}$ reste alors constamment parallèle ou antiparallèle à $O Z$ et s'annule lorsque $\omega_{0}=\omega$. Quelle est alors la forme des courbes de résonance attendue ? Initialement, l'orientation créée dans le système est l'orientation $\mathbf{M}_{0}$ parallèle à $O Z$ (voir Fig. 6). Si la relaxation magnétique est suffisamment intense, sa tendance sera de détruire la composante de $\mathbf{M}_{0}$ sur l'axe $O \mathbf{v}$ perpendiculaire à $O \mathbf{u}$, et donc de réduire l'aimantation à sa composante $M_{u}$ sur $O \mathbf{u}$. Mais cette composante subit alors l'effet du champ efficace $\mathbf{B}_{\text {eff }}$ qui tend constamment à ramener $\mathbf{M}_{u}$ dans une direction soumise à la relaxation ; de fait, si le champ $\mathbf{B}_{\text {eff }}$ est très grand, seule peut subsister la composante de $\mathbf{M}$ parallèle à $O Z$ (les autres composantes oscillant trop rapidement pour subsister en valeur moyenne). On voit donc que l'aimantation $\mathbf{M}$ est soumise aux actions contradictoires de deux processus physiques différents (relaxation magnétique et précession dans le référentiel tournant) réduisant successivement $\mathbf{M}$ à sa composante $M_{u}$, puis $M_{u}$ à sa projection sur $O Z$,

(18) Ceci n'est pas incompatible avec le caractère conservatif du flux du champ magnétique puisque $\Omega_{1 X}$ et $\Omega_{1 Z}$ sont en réalité les composantes de deux champs différents (statique et oscillant).

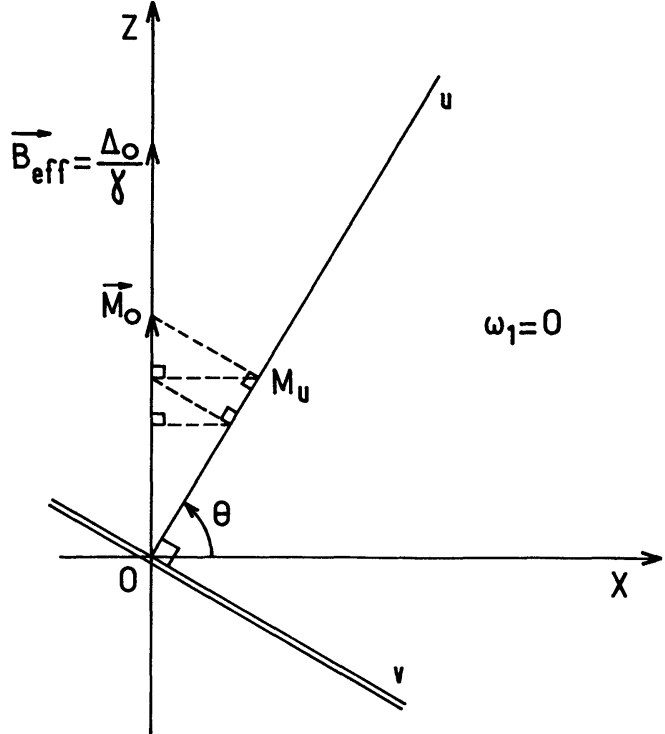

FIG. 6. - Disposition dans le référentiel tournant $O X Y Z$ de l'aimantation initiale $\mathbf{M}_{0}$, de l'axe de non-relaxation magnétique $O \mathbf{u}$, et du plan de relaxation rapide $Y O v$ (perpendiculaire au plan de la figure). Comme on a supposé $\omega_{1}=0$, le champ efficace reste porté par $O Z$ lorsque $\Delta_{0}$ varie et, sous son action conjuguée avec celle de la relaxation, l'aimantation est fortement détruite. Ce n'est qu'à résonance $\left(\Delta_{0}=0\right)$, où $\mathbf{B}_{\mathrm{eff}}=0$, que la projection $M_{u}$ de l'aimantation peut subsister. Ceci explique la courbe fine renversée de la figure 2.

puis projetant à nouveau $M_{u}$ sur $O Z$, etc. Pour finir, l'aimantation qui subsiste est pratiquement nulle : effectivement, en champ inhomogène intense, nous avons trouvé plus haut que $M_{Z}^{\text {s }}$ est négligeable au sommet de la courbe large. Mais nous devons considérer également le cas où $\omega_{0}=\omega$ et où le champ $\mathbf{B}_{\text {eff }}$ s'annule. La situation est alors plus simple, car la relaxation $(R)$ peut imposer sa symétrie en conservant la composante $M_{u}$ de $\mathbf{M}$ sur $O u$; à résonance, l'orientation n'est pas nulle et sa composante sur $O Z$ est simplement celle de $M_{u}$. On comprend donc pourquoi on assiste en champ nul à une reconstruction de l'orientation stationnaire $M_{Z}^{\text {s }}$ (cf. Fig. 2). Notons que le raisonnement géométrique précédent suppose que $O \mathbf{u}$ n'est confondu ni avec $O X$, ni avec $O Z$; nous retrouvons bien que $A_{X Z}$ doit être différent de zéro.

Lorsque $\omega_{1}$ n'est pas nul, la situation est plus complexe car $\mathbf{B}_{\text {eff }}$ change à la fois de longueur et de direction au fur et à mesure que $\omega_{0}$ varie. On constate d'ailleurs facilement (cf. Fig. 7) que deux situations où $\omega_{0}-\omega$ prend des valeurs opposées correspondent à des cas physiques très différents : dans un cas, $\mathbf{B}_{\text {eff }}$ est proche de la direction $O \mathbf{u}$, ce qui entraîne que la composante de $\mathbf{M}$ que $\mathbf{B}_{\text {eff }}$ laisse invariante est peu soumise à la relaxation; dans l'autre cas, $\mathbf{B}_{\text {eff }}$ est proche du plan de relaxation intense et il doit en résulter un effet beaucoup plus notable de la relaxation. On peut donc prévoir que la courbe de résonance donne des valeurs différentes de $M_{Z}^{\mathrm{s}}$ pour deux valeurs opposées de $\omega_{0}-\omega$. On s'attend d'ailleurs à ce que la valeur de $\omega_{0}$ pour laquelle $\mathbf{B}_{\text {eff }}$ 


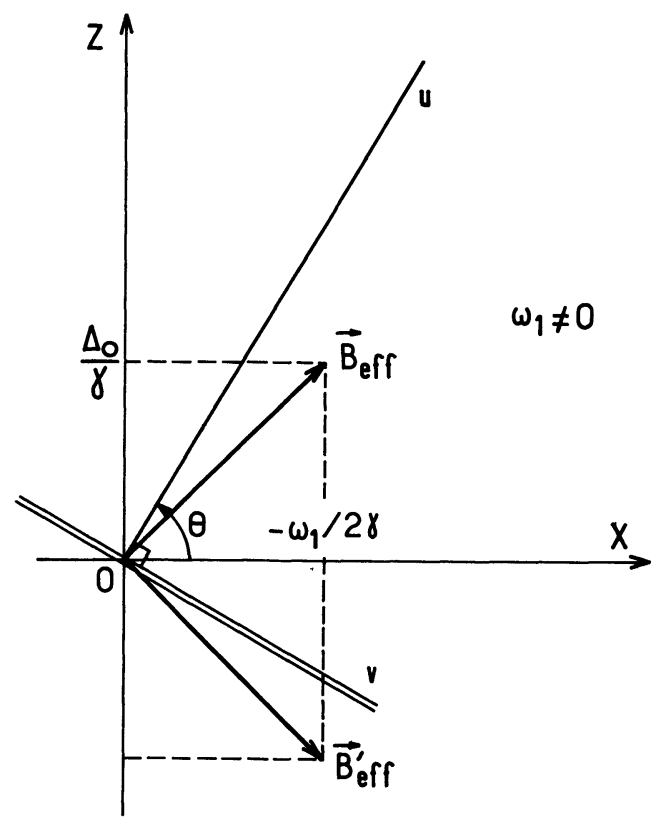

FIG. 7. - Lorsque $\omega_{1} \neq 0$, le champ efficace $\mathbf{B}_{\text {eff }}$ change à la fois de direction et de module lorsque le champ statique $\Delta_{0}$ varie. Deux positions de $\mathbf{B}_{\text {eff }}$ obtenues pour deux valeurs opposées de $\Delta_{0}$ ne sont pas équivalentes : l'une est proche de l'axe de non-relaxation magnétique $O \mathbf{u}$, l'autre du plan de relaxation forte. Dans ce cas, la courbe de résonance est plus compliquée (cf. Fig. 5) et asymétrique.

est porté par Ou joue un rôle particulier ; géométriquement, on calcule facilement que ceci se produit lorsque $\Delta_{0}=\omega_{0}-\omega$ vaut :

$$
\tilde{\Delta}_{0}=\frac{\omega_{1}}{2} \operatorname{tg} \theta=\frac{\omega_{1}}{2} \frac{A_{X Z}}{A_{X X}} .
$$

Lorsque la relaxation due aux inhomogénéités magnétiques est dominante $\left(A_{X X} \gg 1 / T_{1}^{0}\right.$; rappelons que, par hypothèse, $A_{Y Y}$ est ici nul), la comparaison avec (C.18) montre que $\delta \widetilde{\omega} \simeq \widetilde{\Delta}_{0}$, c'est-à-dire que le centre de la courbe fine correspond effectivement à des directions confondues pour $\mathbf{B}_{\text {eff }}$ et $O \mathbf{u}$. Enfin, on voit sur la figure 7 que deux positions du champ efficace correspondant à des valeurs opposées de $\Delta-\tilde{\Delta}_{0}$ ne sont pas équivalentes physiquement; il n'est donc pas étonnant que la courbe fine soit asymétrique et comprenne une courbe de dispersion.

C.2.d Résonance à deux quanta. - Dans les $§ a$, $b$ et $c$ précédents, nous avons supposé que $\omega_{0} \simeq \omega$ et étudié dans ce cas la forme de courbe de résonance obtenue. On peut également se demander ce qui se produit lorsque $\omega_{0} \simeq n \omega$ ( $n$ entier), puisqu'en résonance magnétique classique (champ de radiofréquence homogène) il est bien connu que des effets résonnants existent dans ce cas (transitions à plusieurs quanta). Nous allons nous limiter ici au cas où $\omega_{0} \simeq 2 \omega$ et étudier l'allure des courbes de résonance à deux quanta donnée par un champ linéaire $\left({ }^{19}\right)$ oscillant

$\left({ }^{19}\right)$ Par champ linéaire nous entendons qu'en chaque point $\mathbf{r}$, $\mathbf{B}_{1}(\mathbf{r}, t)$ oscille en fonction du temps en gardant une même direction, cette direction dépendant bien sûr de $\mathbf{r}$. complètement inhomogène (nous supposerons que $\omega_{1}=0$; de plus, nous négligerons ici l'effet des inhomogénéités de champ statique).

Dans (B.7), les termes qui peuvent être responsables des effets cherchés sont $R_{z \pm}$ et $R_{ \pm z}$ et, de façon plus précise, les parties de ces coefficients évoluant comme $\mathrm{e}^{ \pm 2 i \omega t}$. On calcule aisément que ces parties, notées $\hat{R}_{z+}$ et $\hat{R}_{-z}$ valent :

$$
\begin{array}{r}
\hat{R}_{z+}=\sum_{n, l, m} \frac{\left\langle\Omega_{1 z} \mid \varphi_{n, l, m}\right\rangle\left\langle\varphi_{n, l, m} \mid \Omega_{1+}\right\rangle}{4 V} \times \\
\times \mathrm{e}^{2 i \omega t} \frac{\tau_{n, l}}{1+i\left(\omega-\omega_{0}\right) \tau_{n, l}}
\end{array}
$$

et :

$$
\begin{aligned}
& \hat{R}_{-z}=\sum_{n, l, m} \frac{\left\langle\Omega_{1+} \mid \varphi_{n, l, m}\right\rangle\left\langle\varphi_{n, l, m} \mid \Omega_{1 z}\right\rangle}{4 V} \times \\
& \times \mathrm{e}^{-2 i \omega t} \frac{\tau_{n, l}}{1-i \omega \tau_{n, l}} .
\end{aligned}
$$

Les seuls autres coefficients de la matrice $(R)$ qui jouent un rôle important sont les termes diagonaux ; ils donnent un raccourcissement des temps de relaxation transversale et longitudinale, que l'on calcule facilement en faisant $\omega_{0}=2 \omega$ dans (B.27) et (B.36), et que nous supposerons incorporés dans les temps $T_{1}$ et $T_{2}$; de plus, ils donnent un déplacement :

$$
\delta \omega_{0}^{*} \simeq \sum_{n, l, m} \frac{\left|\left\langle\Omega_{1+} \mid \varphi_{n, l, m}\right\rangle\right|^{2}}{6 \omega V} .
$$

Pour finir, les équations d'évolution de

$$
\hat{M}_{+}=M_{+} \mathrm{e}^{-2 i \omega t}
$$

et $M_{z}$ sont :

$$
\left.\begin{array}{r}
\frac{\mathrm{d}}{\mathrm{d} t} \hat{M}_{+}=\left[-\frac{1}{T_{2}}+i\left(\omega_{0}+\delta \omega_{0}^{*}-2 \omega\right)\right] \hat{M}_{+}+ \\
+i \frac{\Omega_{1 z+}}{\omega} M_{z} \\
\frac{\mathrm{d}}{\mathrm{d} t} M_{z}=\frac{i}{2} \frac{\Omega_{1 z+}^{*}}{\omega} \hat{M}_{+}+\text {c. c. }-\frac{1}{T_{1}} M_{z}+\frac{M_{0}}{T_{\mathrm{p}}}
\end{array}\right\}
$$

avec :

$\Omega_{1 z+} \simeq \sum_{n, l, m} \frac{\left\langle\Omega_{1 z} \mid \varphi_{n, l, m}\right\rangle\left\langle\varphi_{n, l, m} \mid \Omega_{1+}\right\rangle}{4 V}$

[nous avons utilisé le fait que $\omega \tau_{n, l} \gg 1$ et $\omega_{0} \simeq 2 \omega$ pour simplifier (C.32a) et (C. 32b)].

Les valeurs stationnaires $\hat{M}_{+}^{\text {s }}$ et $M_{z}^{\text {s }}$ satisfont alors :

$$
\begin{aligned}
& \hat{M}_{+}^{\mathrm{s}}=\frac{i\left(\Omega_{1 z+} / \omega\right) \mathrm{e}^{2 i\left(\omega-\omega_{0}\right) t}}{\left(1 / T_{2}\right)-i\left(\omega_{0}+\delta \omega_{0}^{*}-2 \omega\right)} M_{z}^{\mathrm{s}} \\
& M_{z}^{\mathrm{s}}=M_{0} \frac{T_{1}}{T_{\mathrm{p}}} \times \\
& \times\left\{\begin{array}{r}
1-\frac{\left(\Omega_{1 z+} / \omega\right)^{2} T_{1} / T_{2}}{\left(\omega_{0}+\delta \omega_{0}^{*}-2 \omega\right)^{2}+\left(1 / T_{2}\right)^{2}+} \\
+\left(\Omega_{1 z+} / \omega\right)^{2} T_{1} / T_{2}
\end{array}\right\}
\end{aligned}
$$


La formule (C. 37) appelle quelques remarques. Tout d'abord, on note que la courbe obtenue est lorentzienne, de largeur comparable à $1 / T_{2}$; cette lorentzienne n'existe que si les composantes $\Omega_{1 z}$ et $\Omega_{1+}$ du champ de radiofréquence inhomogène sont simultanément non nulles; la situation rappelle donc celle des résonances habituelles à deux quanta en champ de radiofréquence homogène, où la résonance n'apparaît que si le champ de radiofréquence a simultanément des composantes longitudinale et transversale. On vérifie d'ailleurs bien sur la formule (C.37) que, comme pour un champ homogène, la hauteur de la résonance croît (avant saturation) proportionnellement à la puissance 4 du champ de radiofréquence appliqué. Le fait que les temps de diffusion $\tau_{n, l}$ aient disparu de (C.37) peut se comprendre physiquement : comme nous avons supposé $\omega \tau_{n, l} \gg 1$, chaque atome est soumis à une perturbation magnétique non résonnante dont le spectre est centré en $\omega=\omega_{0} / 2$, et la faible largeur de ce spectre (très éloigné de la fréquence de résonance) ne joue aucun rôle. Par contre, ce que donne la formule (C.37) et qui n'était pas évident a priori, c'est la dépendance des caractéristiques de la résonance (largeur, hauteur) en fonction de la carte du champ inhomogène; on voit en particulier que l'observation de la résonance nécessite l'existence de corrélations entre les perturbations $\Omega_{1 z}$ et $\Omega_{1+}$ vues par chaque atome.

Conclusion. - Les effets étudiés dans cet article de l'inhomogénéité du champ de radiofréquence sur les formes de courbes de résonance peuvent être divers. Ils sont résumés dans le tableau suivant, donnant les formes de courbe dans le cas $\omega_{0} \simeq \omega$ :

TABLEAU

\begin{tabular}{|l|l|l|}
\hline $\begin{array}{c}\text { Champ statique parfaitement ho- } \\
\text { mogène }\end{array}$ & $\begin{array}{l}\text { Champ R. F. totalement inhomo- } \\
\text { gène }\end{array}$ & $\begin{array}{l}\text { Champ R. F. inhomogène compre- } \\
\text { nant une partie homogène }\end{array}$ \\
\hline $\begin{array}{c}\text { Champ statique inhomogène (cor- } \\
\text { rélations entre les différentes } \\
\text { composantes des inhomogénéités) }\end{array}$ & $\begin{array}{l}\text { Courbe large + courbe fine d'ab- } \\
\text { sorption renversée } \\
\text { sorption }\end{array}$ & $\begin{array}{l}\text { Courbe large + courbe fine dépla- } \\
\text { cée, comprenant absorption et dis- } \\
\text { persion }\end{array}$ \\
\hline
\end{tabular}

N.B. : L'observable détectée est l'orientation longitudinale (le pompage étant également longitudinal).

L'étude de la courbe large, qui se présente comme la superposition de plusieurs courbes d'absorption de Lorentz, avec des poids qui dépendent de la carte du champ de radiofréquence inhomogène, permet de mesurer les temps de diffusion des atomes. Ceci fournit une méthode pratique permettant de déterminer expérimentalement les coefficients de diffusion. On pourrait objecter que, sous la forme où nous l'avons étudiée dans cet article, elle nécessite la mesure de la composante longitudinale de l'aimantation, ce qui n'est pas possible dans tous les cas pratiques. En fait, il ne s'agit pas là d'une limitation fondamentale ; au moyen d'un second champ de radiofréquence sonde (comme nous l'avons envisagé au $\S$ C.1.b) ou par des expériences de pulses, on peut facilement mesurer les variations résonnantes des temps $T_{1}^{*}$ ou $T_{2}^{*}$ dues à un champ de radiofréquence inhomogène et en tirer des mesures de coefficients de diffusion. Bien évidemment, toutes ces expériences ne donnent en principe pas d'autres renseignements qu'une étude des effets d'un champ inhomogène statique au voisinage du champ nul. Cependant, d'un point de vue pratique, on sait que les expériences en champs très faibles sont généralement délicates et nécessitent l'utilisation de blindages magnétiques pour bien contrôler la direction du champ homogène statique appliqué sur la cellule ; aussi l'utilisation d'un champ inhomogène oscillant peut-elle être beaucoup plus commode.
On peut d'ailleurs faire la même remarque en ce qui concerne la courbe fine : avec un champ statique inhomogène, on peut par exemple observer une résonance fine renversée comme sur la figure 2 . L'explication théorique de tels effets ne peut être donnée que si on ne néglige pas, comme on le fait habituellement [3] [4] [6], les corrélations entre diverses composantes du champ. Contrairement à la courbe large, la courbe fine ne semble pas pouvoir fournir de renseignements intéressants autres que ceux que donne la résonance magnétique habituelle (en champ homogène). Cependant, la compréhension théorique détaillée des effets des inhomogénéités de la radiofréquence peut être utile dans des expériences de précision, où il est nécessaire de pouvoir évaluer de façon précise leurs conséquences (déformation de la résonance, déplacement de sa position). Tous ces effets ont d'ailleurs été observés expérimentalement dans le cas de ${ }^{3} \mathrm{He}$; la deuxième partie de cet article est consacrée à la description de ces expériences.

Remerciements. - Les auteurs expriment toute leur reconnaissance à $\mathbf{J}$. Dupont-Roc pour de nombreuses discussions qui leur ont été extrêmement utiles, à 'B. Cagnac dont ils ont apprécié l'intérêt amical pour ce travail, et à $\mathrm{C}$. Cohen-Tannoudji qui a bien voulu se charger d'une lecture critique du manuscrit. Ils remercient également J. Seiden d'avoir attiré 
l'attention de l'un d'entre eux, il y a déjà quelques années, sur le fait que la relaxation de noyaux par diffusion dans un champ magnétique inhomogène permettait de mettre en évidence de nombreux modes de diffusion.

\section{Appendice}

MODES DE DIFFUSION DANS UNE SPHÈRE. - Cet appendice est consacré à un rappel d'un certain nombre de formules utiles, relatives aux modes de diffusion dans une sphère.

L'équation de diffusion s'écrit :

$$
\frac{\partial}{\partial t} f(\mathbf{r}, t)=D \Delta f(\mathbf{r}, t)
$$

où $f(\mathbf{r}, t)$ est la densité des atomes étudiés, $D$ leur coefficient de diffusion. Cherchons des solutions de la forme :

$$
f(\mathbf{r}, t)=g_{l m}(t) u_{l m}(r) Y_{l}^{m}(\theta, \varphi)
$$

où $Y_{l}^{m}(\theta, \varphi)$ est l'harmonique sphérique d'ordre $l, m$. Il vient :

$$
\begin{aligned}
& \frac{\mathrm{d}}{\mathrm{d} t} g_{l m}(t)=-\frac{1}{\tau} g_{l m}(t) \\
& D\left[\frac{1}{r} \frac{\mathrm{d}^{2}}{\mathrm{~d} r^{2}} r-\frac{l(l+1)}{r^{2}}\right] u_{l, m}(r)=-\frac{1}{\tau} u_{l, m}(r)
\end{aligned}
$$

où $\tau$ est une constante qui sera précisée par la suite (nous supposerons pour le moment $1 / \tau \neq 0$ ). L'équation $(3 a)$ s'intègre immédiatement :

$$
g_{l m}(t)=\mathrm{e}^{-t / \tau} g_{l m}(0) .
$$

Pour l'équation $(3 b)$, on peut la comparer à l'équation différentielle des fonctions de Bessel sphériques:

$$
\left[\frac{1}{\rho} \frac{\mathrm{d}^{2}}{\mathrm{~d} \rho^{2}} \rho+1-\frac{l(l+1)}{\rho^{2}}\right] f(\rho)=0 .
$$

Parmi les solutions de cette équation différentielle du premier ordre, les solutions qui restent finies pour $\rho=0$ sont les fonctions de Bessel sphériques $j_{l}(\rho)$ [19] dont le comportement à l'origine est en $\rho^{l}$. En posant $r=\rho \sqrt{D \tau}$ on peut rendre identiques les équations $(3 b)$ et $(5)$; par suite, si $u_{l m}(0)<\infty$, on a nécessairement :

$$
u_{l m}(r) \propto j_{l}(\rho=r / \sqrt{D \tau}) .
$$

Reste à choisir une valeur pour $\tau$. Pour cela, nous allons faire intervenir des conditions aux limites : nous supposerons les atomes enfermés dans une cellule sphérique de rayon $R_{0}$ de parois parfaitement réfléchissantes. Le flux d'atomes tombant sur chaque élément infinitésimal de la surface de la sphère est alors exactement compensé par le flux d'atomes réfléchis; par suite, la dérivée normale de $f(\mathbf{r}, t)$ sur la sphère est constamment nulle. Si nous impo- sons cette condition à la fonction (1), il vient :

$$
\left[\frac{\mathrm{d}}{\mathrm{d} r} u_{l m}\right]_{r=R_{0}}=0
$$

c'est-à-dire, compte tenu de (6) :

$$
\frac{R_{0}}{\sqrt{D \tau}}=\beta_{n}^{l}
$$

où $\beta_{n}^{l}$ est le $n$-ième zéro de la dérivée de $j_{l}(\rho)$. Donc, $\tau$ ne peut prendre qu'un ensemble discret de valeurs $\tau_{n, l}[n=1,2,3, \ldots]$ données par :

$$
\tau_{n, l}=\frac{1}{D}\left(\frac{R_{0}}{\beta_{n}^{l}}\right)^{2} .
$$

Pour finir, nous avons un certain nombre de solutions de (1), satisfaisant les conditions aux limites choisies, et dépendant de 3 indices discrets $n$, $l, m$. Chacune de ces solutions décroît exponentiellement dans le temps avec une constante de temps qui dépend de $n$ et de $l$; elle correspond à un mode de diffusion, dont la dépendance en $r$ est donnée par :

$$
\varphi_{n, l, m}(r, \theta, \varphi)=\alpha_{n l} j_{l}\left(\beta_{n}^{l} r / R_{0}\right) Y_{l}^{m}(\theta, \varphi)
$$

où $\alpha_{n l}$ est un facteur de normalisation assurant que l'intégrale dans la sphère de rayon $R_{0}$ de $|\varphi|^{2}$ vaut 1 :

$\alpha_{n l}=\left\{\int_{0}^{R_{0}} r^{2} \mathrm{~d} r j_{l}^{2}\left(\beta_{n}^{l} r / R_{0}\right)\right\}^{-1 / 2}=\frac{1}{\sqrt{R_{0}^{3} Q_{n, l}}}$

avec :

$$
Q_{n, l}=\int_{0}^{1} \rho^{2} \mathrm{~d} \rho j_{l}^{2}\left(\beta_{n}^{l} \rho\right)
$$

Jusqu'ici, nous avons éliminé la possibilité que $\tau$ soit infini c'est-à-dire que les deuxièmes membres des égalités (3) soient nuls. Lorsque c'est le cas, la fonction $g_{l m}(t)$ est constante; quant à $u_{l m}(r)$, elle est donnée par une équation différentielle dont deux solutions indépendantes sont $r^{l}$ et $r^{-l-1}$. Si $l \neq 0$, ces deux solutions doivent être éliminées : la seconde parce qu'elle diverge en $r=0$, la première parce qu'elle ne satisfait pas les conditions aux limites. Si $l=0$, seule la première $\left[u_{00}(r)=\right.$ constante $]$ satisfait ces conditions. Pour finir, le cas $\tau$ infini donne un mode indépendant du temps que nous noterons $\left|\varphi_{0}\right\rangle$, et dont la fonction radiale (normée) est une constante :

$$
\varphi_{0}(r)=\frac{1}{\sqrt{V}}
$$

où $V=4 \pi R_{0}^{3} / 3$ est le volume de la sphère.

Nous admettrons que les fonctions $\varphi_{n, l, m}$ sont orthogonales et qu'elles forment une base pour les fonctions définies dans la sphère et satisfaisant les 
mêmes conditions aux limites qu'elles :

$\left\{\begin{array}{l}\left\langle\varphi_{n, l, m} \mid \varphi_{n^{\prime} l^{\prime} m^{\prime}}\right\rangle=\delta_{n}^{n^{\prime}} \delta_{l}^{l^{\prime}} \delta_{m}^{m^{\prime}} \\ \sum_{n, l, m}\left|\varphi_{n, l, m}\right\rangle\left\langle\varphi_{n, l, m}|+| \varphi_{0}\right\rangle\left\langle\varphi_{0}\right|=1 .\end{array}\right.$

Cherchons alors la solution de (1) qui, à l'instant initial $t=0$, prend en tout point $\mathbf{r}$ une valeur donnée $f_{0}(\mathbf{r})$. Nous avons, d'après $(13 b)$ :

$f_{0}(\mathbf{r})=\sum_{n, l, m}\left\langle\varphi_{n, l, m} \mid f_{0}\right\rangle \varphi_{n, l, m}(\mathbf{r})+\frac{\left\langle\varphi_{0} \mid f_{0}\right\rangle}{\sqrt{V}}$

et la solution cherchée s'écrit :

$$
\begin{aligned}
f(\mathbf{r}, t)=\sum_{n, l, m}\left\langle\varphi_{n, l, m} \mid f_{0}\right\rangle \varphi_{n, l, m}(\mathbf{r}) \mathrm{e}^{-t / \tau_{n, l}}+ \\
+\left\langle\varphi_{0} \mid f_{0}\right\rangle \frac{1}{\sqrt{V}} .
\end{aligned}
$$

Considérons un atome donné, dont la position est $\mathbf{r}^{\prime}$ à un instant $t^{\prime}$; quelle est la probabilité $F(\mathbf{r}, t$; $\left.\mathbf{r}^{\prime}, t^{\prime}\right) d^{3} r$ de le trouver, à un instant $t$ ultérieur, dans l'élément de volume $d^{3} r$ centré en $\mathbf{r}$ ? Lorsque l'atome a subi un grand nombre de collisions aléa- toires, la fonction $F$ obéit à l'équation de diffusion :

$$
\frac{\partial}{\partial t} F=D \Delta_{\mathbf{r}} F
$$

(où $\Delta_{\mathrm{r}}$ désigne le laplacien obtenu par dérivations par rapport à la coordonnée r) ; de plus, on a évidemment :

$$
F\left(\mathbf{r}, t ; \mathbf{r}^{\prime}, t\right)=\delta\left(\mathbf{r}-\mathbf{r}^{\prime}\right) .
$$

Considérée comme une fonction de $\mathbf{r}$ et $t$ dépendant des paramètres $\mathbf{r}^{\prime}$ et $t^{\prime}$, la fonction $F$ est la solution de l'équation de diffusion satisfaisant à la condition initiale d'être égale à $f_{0}(\mathbf{r})=\delta\left(\mathbf{r}-\mathbf{r}^{\prime}\right)$ à l'instant $t^{\prime}$; par suite, en reportant cette valeur de $f_{0}$ dans (15), on obtient :

$$
F\left(\mathbf{r}, t ; \mathbf{r}^{\prime}, t^{\prime}\right)=\frac{1}{V}+\sum_{n, l, m} \varphi_{n, l, m}^{*}\left(\mathbf{r}^{\prime}\right) \varphi_{n, l, m}(\mathbf{r}) \mathrm{e}^{-\left(t-t^{\prime}\right) / \tau_{n, l}}
$$

lorsque $\tau=t-t^{\prime} \gg \tau_{n, l}(\forall n, l), F \simeq 1 / V$ : la probabilité de trouver l'atome en un point quelconque de la cellule est la même.

Donnons pour finir un tableau des valeurs de $\tau_{n, l}$, $\left|I_{n l}\right|^{2}$ [quantité qui intervient dans le développement de $\Omega_{1}$ sur les modes de diffusion, cf. (B.32) et (B.33)] et $Q_{n l}$ [éq. (11)] pour les modes les plus bas :

\begin{tabular}{lccccc} 
& $l=0$ & 1 & 2 & 3 & 4 \\
$n=1$ & - & - & - & - & - \\
$n=2$ & $0,050 R_{0}^{2} / D$ & $0,231 R_{0}^{2} / D$ & $0,090 R_{0}^{2} / D$ & $0,049 R_{0}^{2} / D$ & $0,031 R_{0}^{2} / D$ \\
$n=3$ & $0,017 R_{0}^{2} / D$ & $0,028 R_{0}^{2} / D$ & $0,019 R_{0}^{2} / D$ & $0,014 R_{0}^{2} / D$ & $0,010 R_{0}^{2} / D$ \\
& $0,008 R_{0}^{2} / D$ & $0,011 R_{0}^{2} / D$ & $0,009 R_{0}^{2} / D$ & $0,007 R_{0}^{2} / D$ & $0,006 R_{0}^{2} / D$ \\
\hline$n=1$ & & & $\tau_{n, l}$ & \\
$n=2$ & $32 \times 10^{-11}$ & 0,01014 & 0,00302 & 0,00127 & 0,00064 \\
$n=3$ & $2 \times 10^{-11}$ & $23 \times 10^{-6}$ & $28 \times 10^{-6}$ & $24 \times 10^{-6}$ & $19 \times 10^{-6}$ \\
& & $2 \times 10^{-6}$ & $3 \times 10^{-6}$ & $3 \times 10^{-6}$ & $3 \times 10^{-6}$ \\
\hline$n=1$ & 0,33333 & 0,05123 & 0,02179 & 0,01203 & 0,00755 \\
$n=2$ & 0,02360 & 0,01333 & 0,00865 & 0,00606 & 0,00451 \\
$n=3$ & 0,00824 & 0,00576 & 0,00428 & 0,00331 & 0,00264 \\
& & & $Q_{n l}$ & & \\
\hline
\end{tabular}

On remarque que les modes fondamentaux (modes dont la constante de temps est la plus longue) sont au nombre de trois $(n=l=1 ; m=1,0,-1)$; contrairement à ce qui se produit lorsqu'on prend des conditions aux limites $f=0$ sur la sphère, les modes fondamentaux sont dégénérés et non isotropes $(l \neq 0)$. 


\section{Bibliographie}

[1] Abragam, A., Les principes du magnétisme nucléaire, Bibliothèque des Sciences et techniques nucléaires, (Presses Universitaires de France) 1961.

[2] Bloembergen, N., Purcell, E. M. and Pound, R. V., Phys. Rev. 73 (1948) 679.

[3] Kleppner, D., Goldenberg, H. M. and Ramsey, N. F., Phys. Rev. 126 (1962) 603.

[4] Schearer, L. D., Thèse, Rice University (1966).

[5] Than Myint, M., Thèse, Harvard University (1966).

[6] Gamblin, R. L. and Carver, T. R., Phys. Rev. 138A (1965) 946.

[7] Brenner, D., Phys. Rev. 185 (1969) 26.

[8] Torrey, H. C., Phys. Rev. 76 (1949) 1059.

[9] Solomon, I., C. R. Hebd. Séan. Acad. Sci. 248 (1959) 92.

[10] Solomon, I., Phys. Rev. Lett. 2 (1959) 301.
[11] Torrey, H. C., Phys. Rev. 104 (1956) 563.

[12] Brossel, J., Proceedings of the 3rd Conference on Atomic Physics (1972), Boulder, (Plenum Press), p. 444.

[13] Barbé, R., Leduc, M. et Lalö̈, F., Lettere al Nuovo Cimento 8 (1973) 915.

[14] Novikov, L., Votiakov, S. et Poutirsky, V., C. R. Hebd. Séan. Acad. Sci. 277B (1973) 175.

[15] FA'BRE, C. et DuPONT-Roc, J., communication privée.

[16] Cohen-Tannoudj, C., La relaxation dans les liquides et les gaz, publication interne au laboratoire de l'E. N.S.

[17] Haroche, S., Thèse, Paris (1971); Annls de Phys. 6, 189 et 327.

[18] Broch, F. and Siegert, A., Phys. Rev. 57 (1940) 522.

[19] Voir par exemple : MessiaH, A., Mécanique quantique, Appendice B-II du tome I. 